\title{
On the Formation Dynamics of the North Equatorial Undercurrent
}

\author{
JUNLU LI AND JIANPING GAN \\ Department of Ocean Science and Department of Mathematics, and Center for Ocean Research in Hong Kong and Macau, \\ Hong Kong University of Science and Technology, Hong Kong, China
}

(Manuscript received 21 June 2019, in final form 5 March 2020)

\begin{abstract}
Based on a physics-oriented modeling study, we investigate the underlying forcing processes of the North Equatorial Undercurrent (NEUC). Made up of large-scale ( $\sim 90 \%)$ and mesoscale ( $\sim 10 \%)$ components, the NEUC weakens eastward with a longitude-independent seasonality. The large-scale component reflects the effect of the meridional baroclinic pressure gradient force (PGF_BC). The vertical velocity shear forms the eastward NEUC, when the PGF_BC exceeds the meridional barotropic pressure gradient force (PGF_BT). The mesoscale variability with alternating jets is linked to the wind stress curl in different regions of the tropical North Pacific. Spatially, the NEUC has a northern (NEUC_N) and a southern branch (NEUC_S), which are mainly attributed to the transports from Luzon Undercurrent (LUC) and Mindanao Undercurrent (MUC), respectively. The LUC of $\sim 3 \mathrm{~Sv}\left(1 \mathrm{~Sv} \equiv 10^{6} \mathrm{~m}^{3} \mathrm{~s}^{-1}\right)$ feeds the NEUC_N in summer, while the MUC of $\sim 4$ Sv fuels the NEUC_S in autumn and the two branches do not coexist. The total NEUC transport peaks in August/September, and there exist three distinct periods in a 1-yr cycle: the non-NEUC period in winter, the LUC-driven period in summer, and the MUC-driven period in autumn. Based on the layer-integrated vorticity equation, we diagnose quantitatively that the variation of the NEUC is dominated by the lateral planetary vorticity influx from the LUC and the MUC. These external influxes interact with the internal dynamics of pressure torques and stress curls in the NEUC layer, to jointly govern the NEUC and its variability. Meanwhile, the nonlinearity due to relative vorticity advection near the coast modulates the strength of the NEUC.
\end{abstract}

\section{Introduction}

In the Western Pacific Ocean (WPO), the North Equatorial Current (NEC) bifurcates into the northward Kuroshio (KC) and southward Mindanao Current (MC) (purple arrows in Fig. 1). This NEC-KC-MC (NKM) circulation system, which is located to the east of Philippines, plays an important role in the subtropical water distribution and heat/energy transport in the North Pacific warm pool. Many previous studies have investigated the structures and dominant variabilities of the wind-driven NKM (Kim et al. 2004; Nitani 1972; Qiu and Lukas 1996; Qiu and Chen 2010; Qu and Lukas 2003; Qu et al. 1998; Toole et al. 1988, 1990; Tozuka et al. 2002; Wijffels et al. 1995; Yaremchuk and Qu 2004). However, its undercurrents were not revealed until the 1990s.

The NEC in the upper $500 \mathrm{~m}$ or so carries subtropical warm water westward and feeds into a higher latitude

Corresponding author: Jianping Gan, magan@ust.hk through the KC. Meanwhile, the subsurface current, namely, the North Equatorial Undercurrent (NEUC; Wang et al. 1998), transports intermediate water eastward with a maximum velocity ranging from 0.02 to $0.05 \mathrm{~m} \mathrm{~s}^{-1}$ (Qiu et al. 2013b; Qu et al. 1998; Wang et al. 2015; Yuan et al. 2014; Zhang et al. 2017). Although the NEUC is much weaker than the wind-driven NEC, it substantially affects the transport of the intermediate water across the WPO. The major part of the NEUC is constrained below the bottom of the NEC, and it can reach a depth of $\sim 1500 \mathrm{~m}$. The NEUC has a northern branch and a southern branch, in which the southern one is shallower and stronger due to the northward increasing thickness of the NEC.

The subthermocline undercurrents in the low-latitude region have been broadly investigated (Cravatte et al. 2012; Firing 1987; Gouriou et al. 2006; Philander and Pacanowski 1980; Wang 2005). Subsurface alternating jets were first observed in the tropical region from $3^{\circ} \mathrm{S}$ to $3^{\circ} \mathrm{N}$ (Firing 1987) and from $10^{\circ} \mathrm{S}$ to the equator (Gouriou et al. 2006). Cravatte et al. (2012) found later that the 


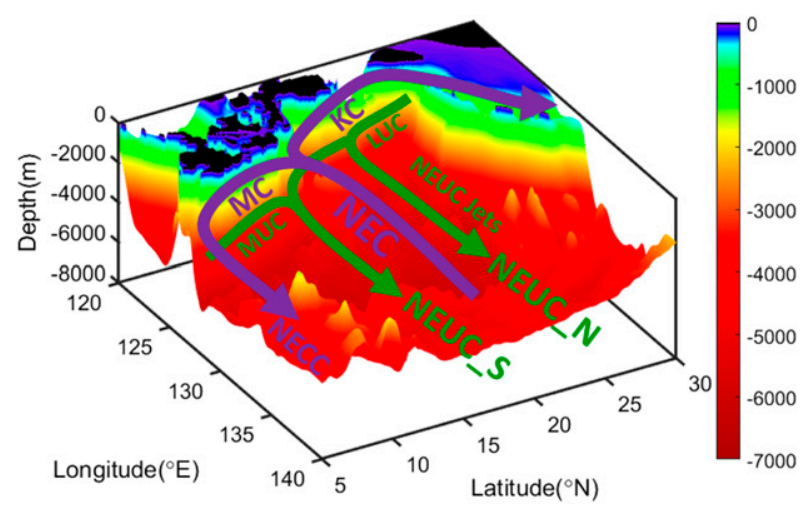

FIG. 1. Schematic of the topography and circulation of the WPO. Purple arrows denote upper-layer currents: the NEC, the $\mathrm{KC}$, the $\mathrm{MC}$, and the NECC. Green arrows denote the undercurrents: the northern (NEUS_N) and southern (NEUC_S) NEUC jets, the LUC, and the MUC. Background color contours represent the bathymetry $(\mathrm{m})$.

alternating subthermocline jets within $10^{\circ} \mathrm{S}-10^{\circ} \mathrm{N}$ were apparent in the WPO and central Pacific but weakened and disappeared approaching the eastern Pacific. However, the generation mechanism of the undercurrents is seldom investigated and remains unclear. Philander and Pacanowski (1980) explained the Equatorial Undercurrent as an extension of the thermocline-trapped wave modes to depths below the wind-driven surface currents. Wang (2005) found that the undercurrents were the result of poleward water movement due to the gain of the planetary vorticity (PV) compensated by the loss of relative vorticity. However, a similar interpretation may not apply to the NEUC in the tropical region.

In the meridional direction, the NEUC exhibits a multicore structure. By adopting the Argo float data, Qiu et al. (2013b) identified the multicore structure of the NEUC, resulting from the long-time mean alternating zonal jets with an amplitude of $\sim 0.1 \mathrm{~m} \mathrm{~s}^{-1}$. The width of each jet ranges from 150 to $200 \mathrm{~km}$. Qiu et al. (2013a) pointed out that the jets were generated by the breaking-down eddies in the wind-induced westward propagating Rossby waves. However, utilizing mooring data, Zhang et al. (2017) found that the zonal velocities in the upper and subthermocline layer were dominated by variabilities with different frequencies, and they suggested that the subthermocline jets could come from subthermocline eddies rather than wind-driven variability. Qiu et al. (2013a) and Zhang et al. (2017) focused on the mesoscale variability and intraseasonal variations in the NEUC. So far, the contribution from the largescale component to the NEUC remains unclear, which is critical to holistically characterizing the NEUC.

In the WPO, two important western boundary undercurrents, the southward Luzon Undercurrent (LUC) and the northward Mindanao Undercurrent (MUC), are closely linked to the NEUC (Fig. 1). The LUC and the MUC were first discovered in the early 1990s using in situ conductivity-temperature-depth (CTD) measurement (Hu and Cui 1989, 1991). This was further confirmed by additional data (Qu et al. 1998) and direct measurements utilizing an acoustic Doppler current profiler (ADCP) deployed at the subsurface (Hu et al. 2013; Zhang et al. 2014). The southward maximum velocity of the LUC ranged from $\sim 7 \mathrm{~cm} \mathrm{~s}^{-1}$ by geostrophic calculation to $27.5 \mathrm{~cm} \mathrm{~s}^{-1}$ by the ADCP, and the mean volume transport was $2-4 \mathrm{~Sv}\left(1 \mathrm{~Sv} \equiv 10^{6} \mathrm{~m}^{3} \mathrm{~s}^{-1}\right)$. The maximum velocity of the MUC was larger than that of the LUC, reaching 10-20 $\mathrm{cm} \mathrm{s}^{-1}$ (Hu et al. 1991; Zhang et al. 2014), and the transport was 8-22 Sv (Hu et al. 1991). Previous studies of the LUC and the MUC were mainly conducted independently, and their connections to the NEUC have not been adequately explored. Apart from the mean southward (northward) water transport, the LUC (MUC) also exhibited a significant intraseasonal variability with a period of 70-80 (60-80) days (Hu et al. 2013; Zhang et al. 2014). Due to a shortage of in situ measurements, we still lack a comprehensive understanding of the seasonality in the LUC and the MUC.

The historical hydrographic data and Argo float data have unveiled general features of the NEUC. The in situ ADCP measurements, on the other hand, have provided a glimpse into the characteristics of the LUC and MUC. Yet, they are not sufficient to provide a holistic understanding of the NKM undercurrent system (NKMU) of LUC, MUC, and NEUC in the WPO, especially its spatiotemporal variability. Moreover, the forcing mechanism such as role of the lateral and vertical forces in the NKMU, which cannot be quantitatively resolved by observations, is still unknown. In addition, the dynamic connections between the NEUC, the LUC, and the MUC remains unclear.

By utilizing a three-dimensional time-dependent, eddyresolving model with idealized coastline and climatological mean forcing, we conduct a process-oriented study to investigate the dynamic features in the NKMU. We investigate the characteristics and dominant forcing mechanism of the NEUC and examine the dynamic connections among the undercurrents, and between the NKM and the NKMU. This study elevates our understanding of the characteristics and underlying physics of the WPO undercurrent system.

\section{Ocean model}

We utilized the Regional Ocean Modeling System (ROMS; Shchepetkin and McWilliams 2005) to conduct a process-oriented study to investigate the NEUC. The ROMS is a three-dimensional, time-dependent circulation 

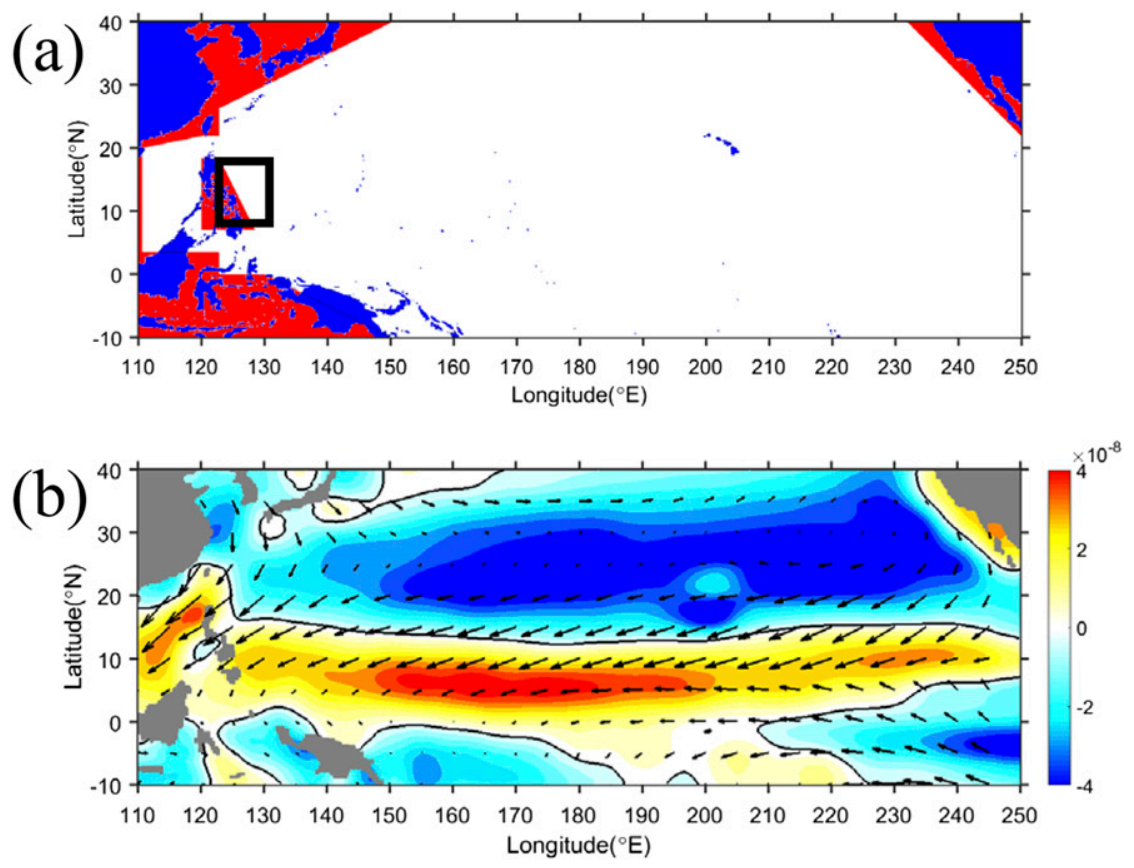

FIG. 2. (a) Realistic coastline (blue) and geometry of the idealized model domain (red). The black box is the NKM/NKMU region of focus in this study. (b) The annual mean wind stress vectors and wind stress curl $\left(\mathrm{Pa} \mathrm{m}^{-1}\right.$; color shaded).

model governed by hydrostatic primitive equations. A local closure scheme based on the level-2.5 turbulent kinetic energy equations (Mellor and Yamada 1982) was used in vertical mixing parameterization. The model domain covered the low-latitude region of the Pacific and the South China Sea (SCS), extending from $110^{\circ} \mathrm{E}$, $10^{\circ} \mathrm{S}$ in the southwest corner to $110^{\circ} \mathrm{W}, 40^{\circ} \mathrm{N}$ in the northeast corner (Fig. 2a). This computational domain provided a high degree of dynamic freedom for the circulation in the NKM/NKMU region (black box in Fig. 2a). To better isolate the physical process in the complex NKM/NKMU, we adopted coastlines with an idealized geometry but preserved the spatial scale and dominant characteristics of the Pacific basin and the SCS to identify the underlying formation processes for the NEUC as well as their unknown connections with the undercurrents in the western boundary.

The NKM and the NKMU systems exist mainly in the upper $2000 \mathrm{~m}$ of the ocean, thus the water depth was set to a constant of $4000 \mathrm{~m}$. The Pacific basin connects the marginal SCS with two straits, the Luzon Strait (LS) at $\sim 20^{\circ} \mathrm{N}$ and the Mindoro Strait (MS) at $\sim 5^{\circ} \mathrm{N}$ (Fig. 2a). Trigonometric-function-fitting bathymetry was applied in the LS, which enabled a reasonable water exchange through the LS. Meanwhile, a shallower topography $(450 \mathrm{~m})$ was adopted in the MS. The model adopted a uniform horizontal rectangular grid with a grid size of $11 \mathrm{~km}$. We adopted 30-level stretched generalized terrain- following coordinates with a higher vertical resolution in the upper water column to better resolve the NEC/NEUC which was the focus of this study.

We forced the model with wind stress derived from climatological (averaged from 1988 to 2010) monthly blended $0.25^{\circ}$ sea surface winds released by the National Oceanic and Atmospheric Administration (NOAA) and the National Climatic Data Center (NCDC; https:// www.ncdc.noaa.gov/data-access/marineocean-data/blendedglobal/blended-sea-winds), based on the bulk formulation (Fairall et al. 2003). The annual mean wind stress and wind stress curl (WSC) are shown in Fig. 2b. The model was also forced by atmospheric heat and salt fluxes from climatological monthly reanalysis data released by the National Centers for Environmental Prediction (NCEP).

We applied the radiation boundary condition (Raymond and Kuo 1984) at the northern and southern open boundaries of the domain for the velocity and tracers, and the no-gradient boundary condition is applied to the sea surface elevation along the open boundaries. The annual mean temperature $T$ and salinity $S$ from World Ocean Atlas 2013 (WOA13), and zero velocities and elevation were used to initialize the model. In this processoriented study, we adopted domain and horizontally averaged $T$ and $S$ profiles as the initial condition to extract the dominant process invoked in the NEUC. The horizontal homogeneous initial condition may suppress the effect of baroclinic instability during the first few 
years, however, the circulation pattern of NEUC is not overly sensitive to the initial $T$ and $S$ horizontal gradients. The model was spun up for 50 years, even though the NEUC jets, LUC, and MUC have all reached quasisteady states after 20 years. We used the model variables in the last 10 years of the 50 -yr run to conduct analyses in this study. The idealized model was designed with physically sensible resolution and domain, validated atmospheric fluxes and simplified topography to facilitate the process-oriented study.

\section{Characteristics of the NEUC}

\section{a. General flow pattern}

The subtropical highs to the east of Taiwan and the Mindanao Dome (MD) to the southeast of Mindanao Island with distinct sea surface height ( $\mathrm{SSH}$ ) captured by the model reflect the general circulation pattern in the region, and they are consistent to those derived from the Archiving, Validation and Interpretation of Satellite Oceanographic Data (AVISO; Figs. 3c,d). The observed westward NEC, eastward North Equatorial Countercurrent (NECC) and coherent western boundary currents of $\mathrm{KC}$ and $\mathrm{MC}$, are also reasonably reproduced (Figs. 3a,b), both qualitatively and quantitatively. As compared with the realistic model, the NECC locates relatively south mainly due to the missing of Halmahera Island in the idealized coastline (Fig. 3b). This, however, has limited effect on the focused undercurrent system. Furthermore, both the magnitude and phase of the seasonal variation of the NKM transport are comparable to those calculated from in situ observations, historical hydrographic data and numerical models (Fig. 4d) (Jensen 2011; Qiu and Lukas 1996; Qu et al. 1998, 2012; Yaremchuk and Qu 2004).

Figure 4a shows that the NEC is mainly trapped in the upper $\sim 500 \mathrm{~m}$ in the subtropical region of the North Pacific from $6^{\circ} \mathrm{N}$ to higher than $20^{\circ} \mathrm{N}$. The annual mean volume transport of the NEC is $\sim 50 \mathrm{~Sv}$ (Fig. $4 \mathrm{~d}$ ), which is comparable to those reported in previous studies ranging from 40 to $70 \mathrm{~Sv}$ (Nitani 1972; Qu et al. 1998; Toole et al. 1988, 1990; Yaremchuk and Qu 2004). Vertically, the model is capable of producing not only the wind-driven upper-layer currents, but also the undercurrents: the NEUC, LUC, and MUC (Figs. 4a-c). All the currents and undercurrents generated by our model have reasonable widths, depths and intensities as compared to previous studies (Qu et al. 1998; Wang et al. 2015). The vertical structures of the upper currents/undercurrents are consistent with the ADCP mooring observations (Hu et al. 2013; Ren et al. 2018; Zhang et al. 2014, 2017). The northward weakening and deepening of the NEUC jets are clearly revealed (Fig. 4a). The features that the MUC is stronger than the LUC, and the core of the MUC is shallower than that of the LUC (Figs. 4b,c), have also been identified by previous studies ( $\mathrm{Qu}$ et al. 1998; Wang et al. 2015).

Similar to the results of Qiu et al. (2013b), the major parts of the undercurrents are trapped between the isopycnal surfaces equal to 26.8 and $27.6 \mathrm{~kg} \mathrm{~m}^{-3}$ (green lines in Figs. 4a-c), namely, the NKMU layer. Thus, the undercurrent transport in this study is defined as the net integrated-velocity within the NKMU layer. Similarly, the upper-layer current transport is defined as the net integrated velocity between the sea surface to the isopycnal surface of $26.8 \mathrm{~kg} \mathrm{~m}^{-3}$, which is named the NKM layer. The NEUC is a basin-scale current and exhibits multiple-core structure (Fig. 5). Alternating jets exist in the NEUC, in which there are two significant eastward jets, namely northern (NEUC_N) and southern (NEUC_S) NEUC (Fig. 5b). The NEUC_S is shallower than the NEUC_N and its velocity core locates at $\sim 600 \mathrm{~m}$ (Fig. 4a). At $1000 \mathrm{~m}$, the NEUC_S becomes weaker and the NEUC_N becomes significant (Fig. 5c). The multiple NEUC jet phenomenon and their poleward deepening are also shown by the geostrophic velocity derived from observational temperature and salinity profiles (Qiu et al. 2013b; Wang et al. 2015; Yuan et al. 2014). Although the alternating NEUC jets have been confirmed by many observations and model simulations, their widths, strengths, and the number of the jets below the NEC remain uncertain. Qiu et al. (2013b) utilized Argo float data to show 3 distinct NEUC jets locating at $9^{\circ}, 13^{\circ}$, and $18^{\circ} \mathrm{N}$. However, combining the Argo float, World Ocean database 2009 and CTD XBT temperature data, Wang et al. (2015) discovered only two patches of eastward velocity in the NEUC from $8^{\circ}$ to $18^{\circ} \mathrm{N}$. Qiu et al. (2013a) adopted the OGCM for the Earth Simulator (OFES) to reproduce three NEUC jets, with intensities smaller than the observations. The Global Hybrid Coordinate Ocean Model (HYCOM) reproduces the NEUC jets with smaller width and weaker strength comparing to OFES (not shown here).

Our results show that the NEUC jets extend below a depth of $1500 \mathrm{~m}$, which is also consistent with the observations (Qu et al. 1998; Yuan et al. 2014; Zhai et al. 2013). The maximum volume transport of the NEUC between $8^{\circ}$ and $18^{\circ} \mathrm{N}$ in a year is $\sim 9 \mathrm{~Sv}$ in the western and central tropical Pacific and decreases eastward, which is much weaker than that of the NEC (Fig. 6). Clearly, our process-oriented model captures well the dominant features and pattern of the NEUC for us to conduct investigation of the undefined origin of the NEUC and their connection with the western boundary undercurrents. 

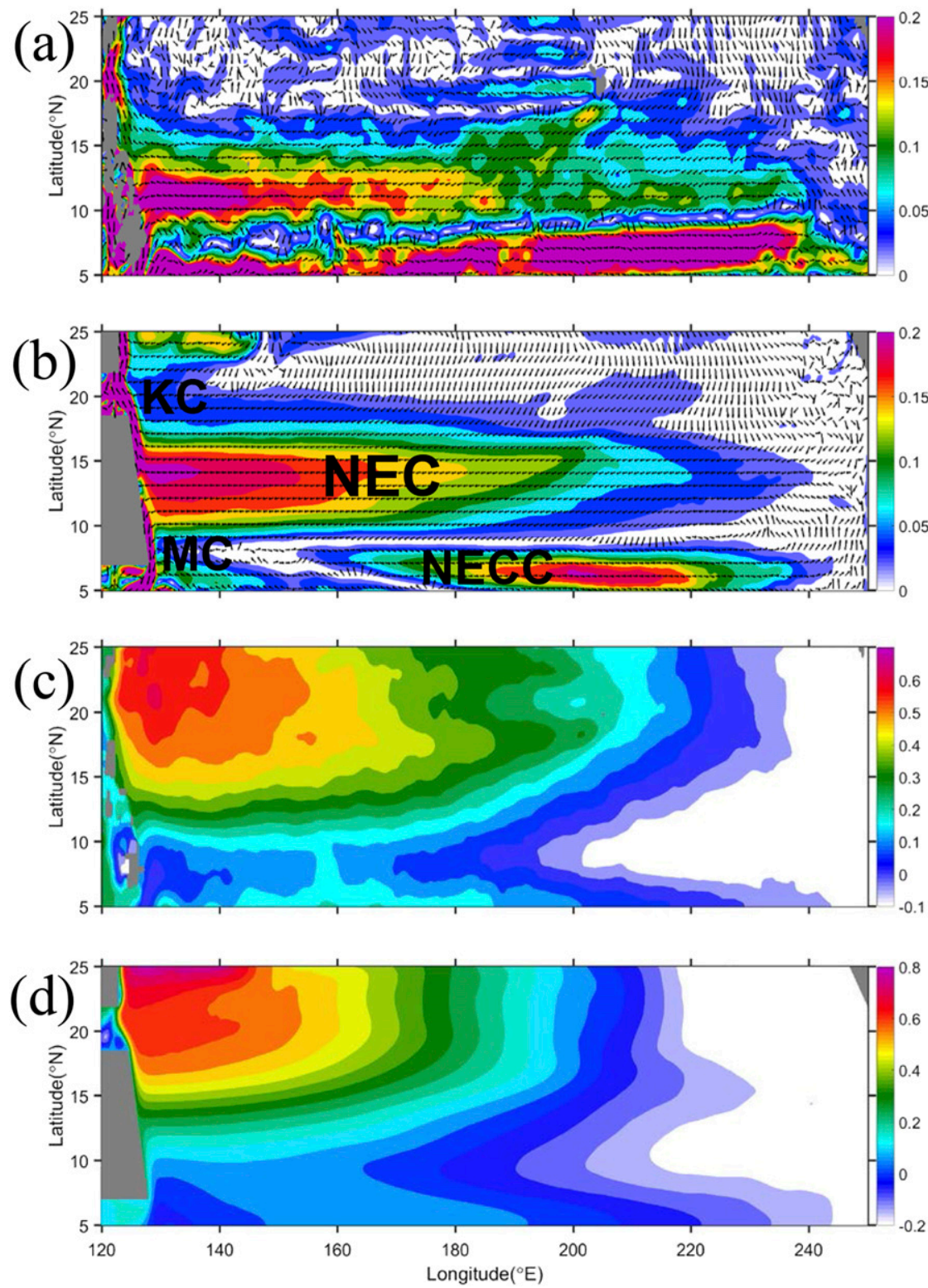

FIG. 3. Surface velocity magnitude ( $\mathrm{m} \mathrm{s}^{-1}$; color contours) and direction (vectors) adopted from (a) AVISO and (b) the idealized model. SSH (m; color contours) adopted from (c) AVISO and (d) the idealized model.

The seasonality of the NEC is longitude dependent, since the transport is affected by both local wind forcing and remote effects caused by the westward propagating Rossby waves (Qiu and Lukas 1996). Generally, seasonal variation is the dominant feature of the NEC transport in the WPO $\left(130^{\circ}\right.$ $\left.150^{\circ} \mathrm{E}\right)$ and the eastern Pacific Ocean (EPO, $150^{\circ}-$ $110^{\circ} \mathrm{W}$ ), and a semiannual variation prevails in the central Pacific Ocean (CPO; $150^{\circ} \mathrm{E}-150^{\circ} \mathrm{W}$ ) (Fig. 6a). The pattern of the NEC transport as a function of time and longitude is highly correlated with the SSH and WSC fields, suggesting that it is dominated by barotropic effects.

Unlike the NEC transport, the seasonality of the NEUC transport is longitude independent. The NEUC appears only in summer and autumn, and is replaced by a westward transport in winter and spring (Fig. 6b). We utilize the horizontal transport streamfunction integrated within the NKMU layer to illustrate the annual cycle of the NEUC (Fig. 7). The meridional gradient of streamfunctions $\Psi$ indicates the zonal transport: $U_{\mathrm{NEUC}}=-\partial \Psi / \partial y$, in which $y$ is the meridional axis 

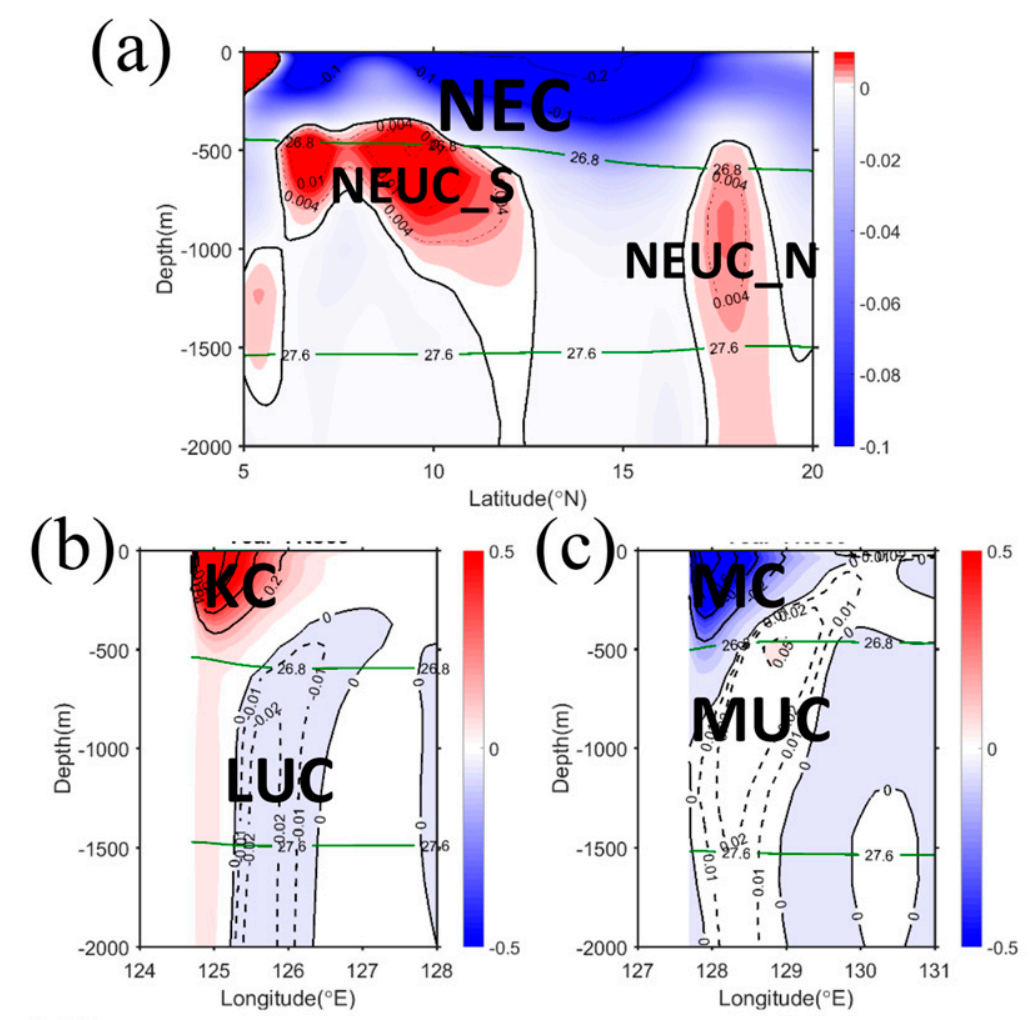

(d)
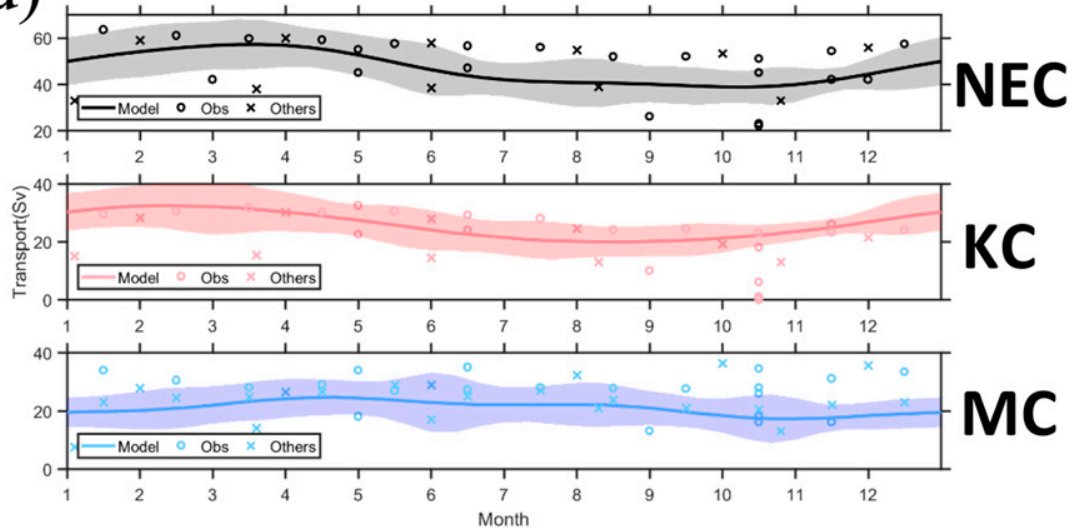

FIG. 4. Cross-sectional velocity $\left(\mathrm{m} \mathrm{s}^{-1}\right)$ in (a) the NEC/NEUC $\left(130^{\circ} \mathrm{E}\right)$, (b) the $\mathrm{KC} / \mathrm{LUC}$ $\left(18^{\circ} \mathrm{N}\right)$, and $(\mathrm{c})$ the MC/MUC $\left(8^{\circ} \mathrm{N}\right)$ sections. Positive values denote northward or eastward velocity. Green lines denote the top and bottom isopycnal surfaces of the NKMU layer. (d) Seasonal variation of the $\mathrm{NEC}$, the $\mathrm{KC}$, and the MC volume transport (Sv) integrated above the isopycnal surface of potential density $\left(\mathrm{kg} \mathrm{m}^{-3}\right)$ equal to 26.8 adopted from the idealized model (lines), and transport with different definitions in previous observations (circles) and models (crosses). The shaded region shows the standard deviation of the idealized model result.

and the larger $\Psi$ values are on the right-hand side of the flow direction. In spring, a westward transport prevails in the entire region, which suggests the absence of the eastward NEUC in this season (dashed arrow in Fig. 7a). A relatively weak eastward NEUC appears in summer (Fig. 7b), which is located mainly in the northern NKMU region. In autumn, the NEUC becomes stronger and its core moves to the southern NKMU region (Fig. 7c). After autumn, the NEUC weakens and is eventually replaced by a strong westward current in winter (Fig. 7d). The evolution of the NEUC reveals its important meridional asymmetric structure, in which the NEUC_N (NEUC_S) is more significant during summer (autumn). The meridional asymmetricity suggests that the 

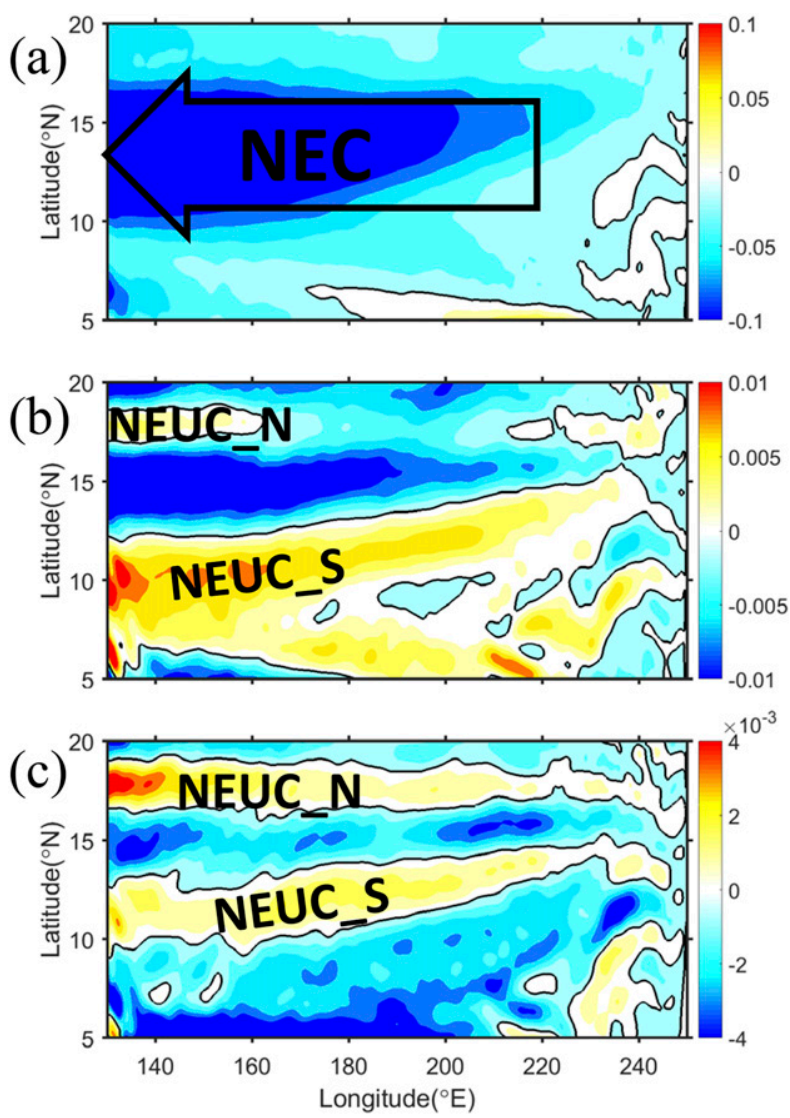

FIG. 5. Annual mean zonal velocities $\left(\mathrm{m} \mathrm{s}^{-1}\right)$ at water depths of (a) 200, (b) 600, and (c) $1000 \mathrm{~m}$. NEUC_S refers to the southern and NEUC_N to the northern branch of the NEUC. Positive values denote eastward velocity.

NEUC may have northern and southern origins associated with the two western boundary undercurrents (LUC and MUC) in the WPO in different periods. These will be discussed in more details in section 4 .

\section{b. Large-scale and mesoscale components}

Qiu et al. (2013a) utilized a filter to isolate currents of different scales in the depth-independent alternating jets. After removing the large-scale $(>400 \mathrm{~km})$ zonal velocity signals by applying a $4^{\circ}$-box moving-average high-pass filter, they found that the alternating jets extended from the sea surface to $\sim 2000 \mathrm{~m}$ below. The $4^{\circ}$ box was chosen due to the jet width.

We also find that the alternating jets are independent of the background large-scale flows. By using the $4^{\circ}$-box high-pass filter, the NEUC is decomposed into largescale $(>400 \mathrm{~km})$ and mesoscale $(<400 \mathrm{~km})$ components (Fig. 8). Different from the previous study (Qiu et al. 2013a), we further quantify the contributions of the variabilities at these two scales to the subsurface net transport within the NKMU layer. The velocity
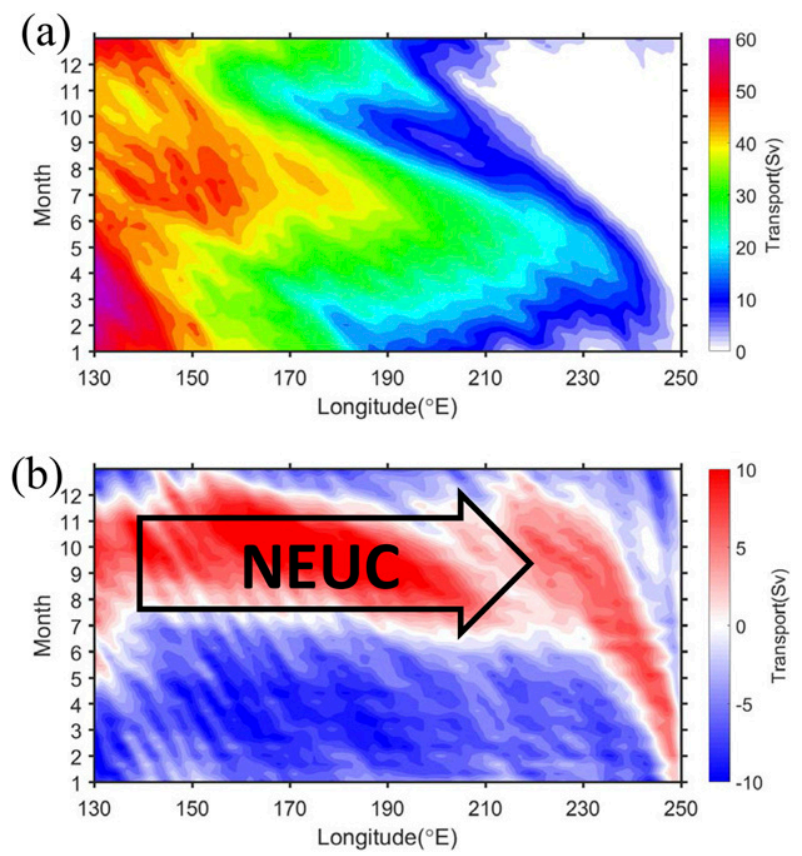

FIG. 6. Longitude-dependent seasonal variation of (a) the NEC and (b) the NEUC volume transport (Sv). The NEC is defined as the depth-integrated zonal velocity from the sea surface to the isopycnal surface of the potential density $\left(\mathrm{kg} \mathrm{m}^{-3}\right)$ equals to 26.8 vertically, from $8^{\circ}$ to $18^{\circ} \mathrm{N}$ meridionally, and positive values denote westward transport. The NEUC is defined as the depth-integrated zonal velocity between the isopycnal surfaces of the potential densities $\left(\mathrm{kg} \mathrm{m}^{-3}\right)$ equal to 26.8 and 27.6 vertically, from $8^{\circ}$ to $18^{\circ} \mathrm{N}$ meridionally, and positive values denote eastward transport.

averaged over $130^{\circ}-135^{\circ} \mathrm{E}$ is used to represent the NEC/NEUC velocity profile in the WPO. For the EPO section, the velocity is averaged over $140^{\circ}-135^{\circ} \mathrm{W}$. The velocities at both scales weaken with depth (Figs. 8a-c). The large-scale component of the NEUC is wide and mostly flows eastward, mainly contributing to the eastward transport of intermediate water (Fig. 8b). However, the mesoscale component shows that the neighboring westward jets (Figs. 8c,f) compensate for the eastward jets, thus their combined effect on the net subsurface transport in the NKMU layer is limited.

To better quantify the contributions of the two components to the net subsurface transport of the NEUC, we use the model output in the last 10 years to illustrate the longitude-dependent variability of the NEUC net transport at the large and mesoscales. Figure 9 shows that the large-scale and mesoscale components account for $\sim 90 \%$ and $\sim 10 \%$ of the total NEUC net transport, respectively. Both components weaken eastward, except for the relatively strong mesoscale variation in the EPO, which is related to the high-frequency variability near the eastern boundary generated by local wind (Qiu et al. 2013a; Yoshida et al. 2010). The large-scale component 
(a)

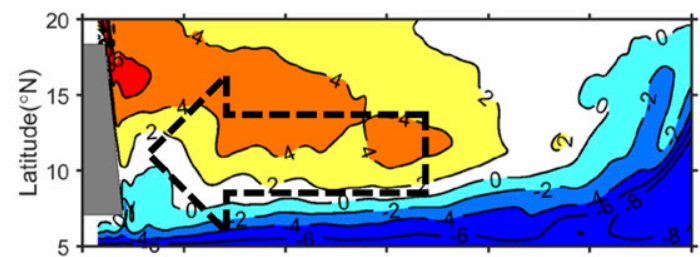

(b)

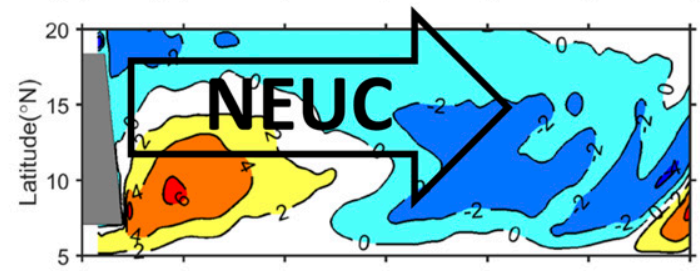

(c)

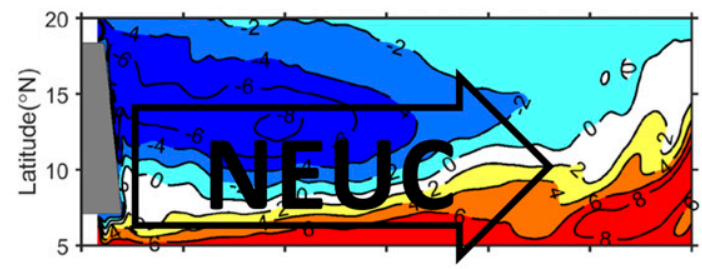

(d)

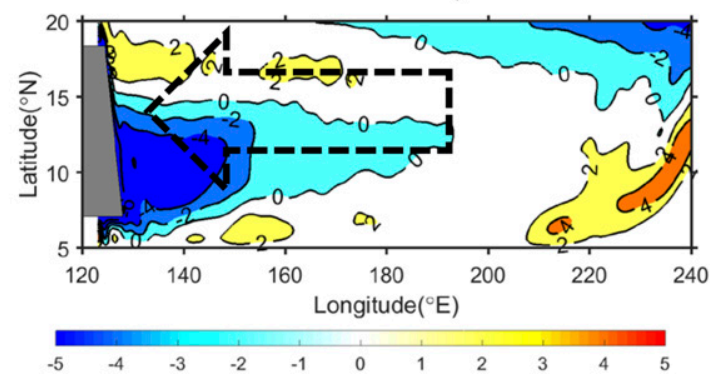

FIG. 7. Seasonal transport streamfunction $(\Psi ; \mathrm{m} \mathrm{Sv})$ integrated between the isopycnal surfaces of the potential densities $\left(\mathrm{kg} \mathrm{m}^{-3}\right)$ equal to 26.8 and 27.6 in (a) spring, (b) summer, (c) autumn, and (d) winter. The meridional gradient of $(-\partial \Psi / \partial y)$, indicates the transport of the NEUC in which the larger $\Psi$ values appear to the right of the direction of flow.

exhibits significant seasonal variation at all longitudes, while the mesoscale component is dominated by intraseasonal variability. As a result, the large-scale component is significantly larger than the mesoscale component in terms of both magnitude and variability. We have also conducted sensitivity tests using a filter with a $6^{\circ}$ box, and the results are robust.

Apparently, the NEUC exhibits different characteristics and variabilities at different longitudes (Fig. 9). Thus, we have extended our understanding of the NEUC in three isolated regions of the tropical North Pacific.

\section{c. Barotropic and baroclinic components}

The large-scale component of the NEUC weakens eastward, with a seasonality that is independent of longitude. In addition, the spatiotemporal pattern of the mesoscale component indicates a strong intraseasonal variation in the WPO, a weak variability in the CPO, and seasonality in the EPO (Fig. 9b). To investigate the underlying mechanisms, we first separate the tropical North Pacific into three regions according to the spatiotemporal pattern of the mesoscale component of the NEUC. We choose the NEUC transport at $130^{\circ} \mathrm{E}, 180^{\circ}$, and $140^{\circ} \mathrm{W}$ to represent the typical variabilities in the WPO, CPO, and EPO regions, respectively. In the WPO, CPO, and EPO, the eastward NEUC volume transport varies from -10 to $9 \mathrm{~Sv}$, from -8 to $11 \mathrm{~Sv}$, and from -5 to $5 \mathrm{~Sv}$, respectively (Figs. 10a,c,e). Clearly, the NEUC weakens toward the east because the pink areas become smaller from WPO to CPO to EPO (Figs. 10a,c,e). The seasonal phases in these regions all exhibit an eastward transport peaking in August/September and a westward transport peaking in February/March.

The NEUC is mainly a geostrophic current. Thus, the meridional pressure gradient force (PGF) is the dominant term balancing the Coriolis term due to the water movement. In this study, we decompose the PGF into two components. The first component is generated by the gradient of the sea surface elevation, which is named barotropic PGF (PGF_BT) because it is depth independent. The second component is caused by the gradient of the density, which is named baroclinic PGF (PGF_BC) due to its depth-dependent feature. The PGF can be shown as

$$
\underbrace{-\frac{1}{\rho_{0}} P_{y}}_{\text {PGF }}=\underbrace{-\eta_{y} g}_{\text {PGF_BT }} \underbrace{-\frac{g}{\rho_{0}} \int_{-H}^{\eta} \rho_{y} d z}_{\text {PGF_BC }},
$$

where $P_{y}$ is the meridional PGF and $\eta_{y}$ and $\rho_{y}$ are the meridional gradient of the SSH and density, respectively. PGF_BT and PGF_BC have opposite signs. To describe the dynamics of PGF, we change the sign of PGF_BC. The negative PGF_BC represents the baroclinic effect sustaining the eastward NEUC, while the negative PGF_BT indicates the barotropic effect restraining the eastward velocity. Thus, the residual part (pink shaded area in the right panel of Fig. 10) denotes the favorable PGF for the NEUC, when PGF_BC exceeds PGF_BT. The eastward NEUC only exists when the PGF_BC overcomes the PGF_BT, which is consistent with the conclusion in earlier study that the slope of the pycnoclines should be strong enough to provide necessary condition for the existence of the undercurrents (Wang and $\mathrm{Hu}$ 1999). Apparently, the eastward-weakening NEUC transport is well correlated with the weakened PGF_BT, PGF_BC as well as their residual part (right panel of Fig. 10), because the pink areas in Fig. 10 become smaller from the WPO to EPO.

In the WPO and EPO, both PGF_BT and PGF_BC govern the seasonal variation of the NEUC (Figs. 10b,f). However, a semiannual PGF controls the NEUC seasonal variation in the CPO region (Fig. 10d). The semiannual variation of the $\mathrm{SSH}$ gradient may be 


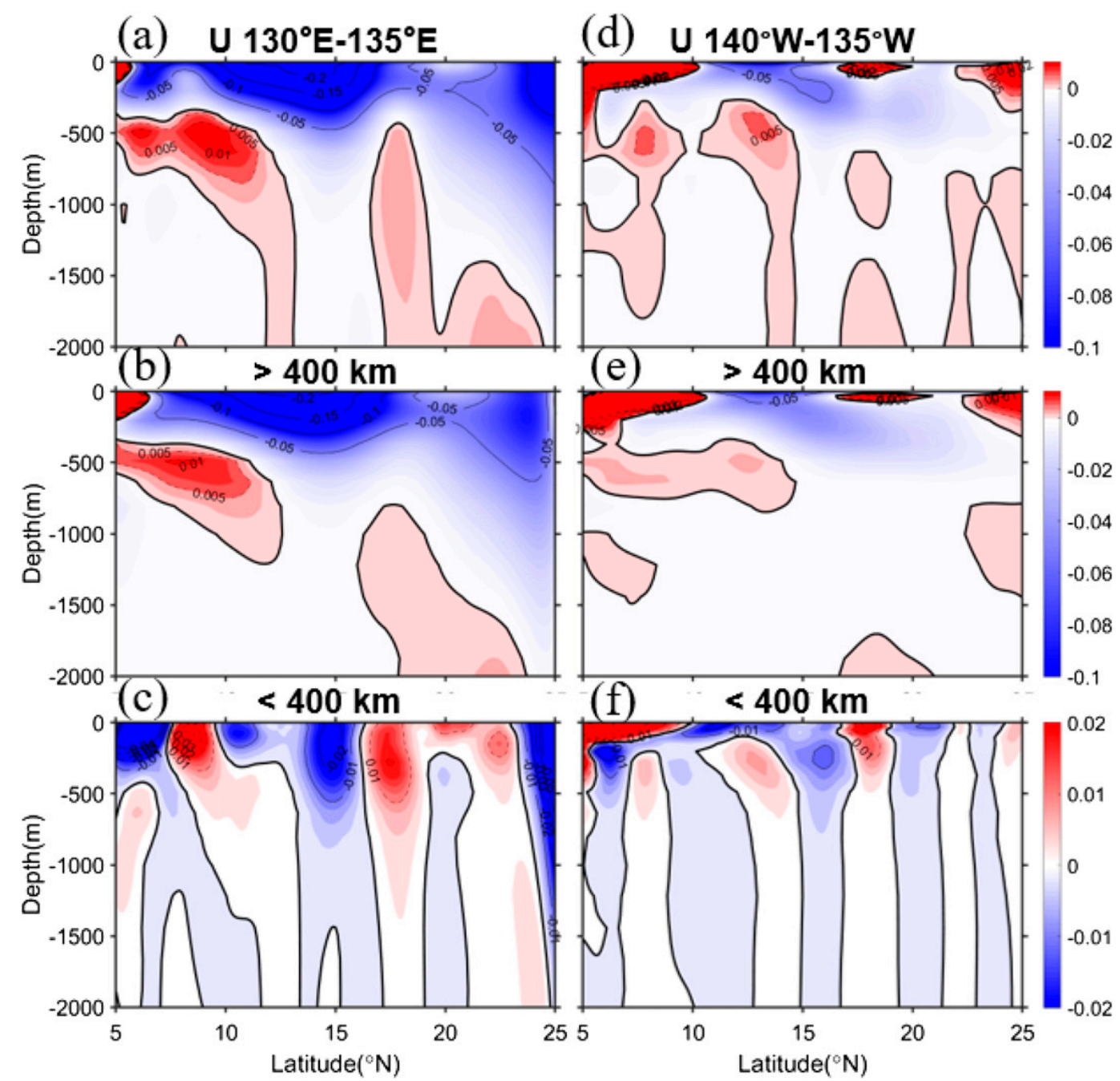

FIG. 8. (a) Total, (b) large-scale, and (c) mesoscale components of the zonal velocity ( $\mathrm{m} \mathrm{s}^{-1}$ ) profiles averaged over $130^{\circ}-135^{\circ} \mathrm{E}$. (d)-(f) As in (a)-(c), but averaged over $140^{\circ}-135^{\circ} \mathrm{W}$. The large-scale and mesoscale components are defined by the meridional length scale of the zonal velocity larger and smaller than $400 \mathrm{~km}$, respectively. Positive values (red) denote eastward velocity. The interval of the colored contours is $0.0025 \mathrm{~m} \mathrm{~s}^{-1}$.

related to the Hawaii Island-induced WSC (Sakamoto et al. 2004; Xie et al. 2001; Yoshida et al. 2010). The seasonal/semiannual/seasonal variation structure of PGF_BT is well correlated with the intensity variation of the NEC in the WPO/CPO/EPO (Fig. 6a), and they are likely induced by the spatially correlated WSC in the tropical North Pacific (not shown here). Once a higher $\mathrm{SSH}$ is formed, the dome of SSH deepens the isopycnals in the upper several hundreds of meters of the water column. Thus, the variation of PGF_BC reflecting the tilting of the isopycnals is correlated with the change in the $\mathrm{SSH}$ gradient.

However, we also found that the WSC field cannot explain the similar period of emergence of the NEUC in the WPO, CPO and EPO. The NEUC in summer to autumn is dependent of the seasonal phases of the combined PGF_BT and PGF_BC effect, which acts dynamically to sustain the NEUC and its seasonal variation. In addition, external forcings from influxes of the LUC and MUC should play significant roles, besides the internal dynamic hub for the formation and sustenance of the NEUC, as will be shown below.

\section{Dynamic linkage among the undercurrents}

Seasonal variation of the NEUC transport in Figs. 7 and 10 indicates a linkage among the undercurrents in the WPO. We use the vectors of the depth-integrated velocity to identify the flow pattern in NKMU region. Three typical periods of the NEUC, namely, the non-NEUC 


\section{(a) Large-scale}

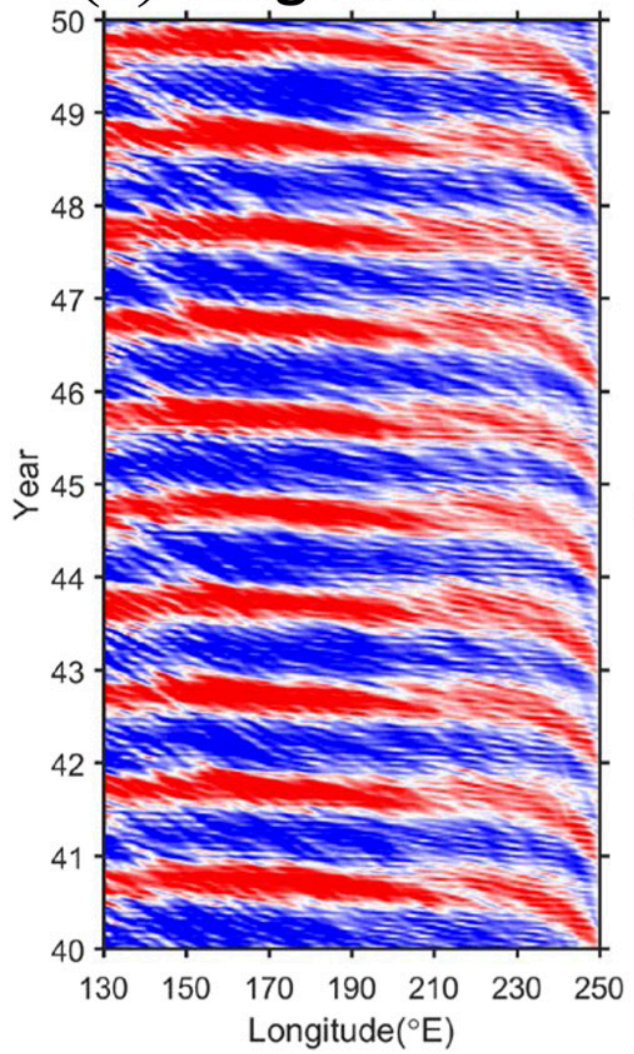

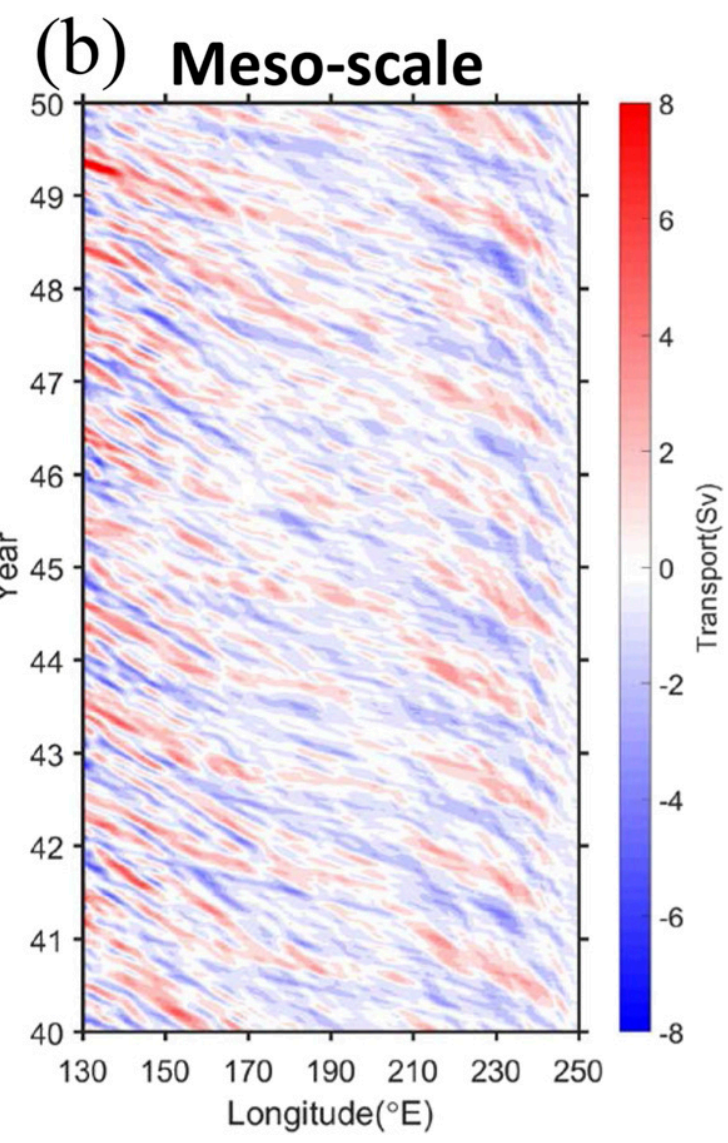

FIG. 9. (a) Large and (b) mesoscale components of the NEUC net transport (Sv) integrated between the isopycnal surfaces of the potential densities $\left(\mathrm{kg} \mathrm{m}^{-3}\right)$ equal to 26.8 and 27.6 vertically, from $8^{\circ}$ to $18^{\circ} \mathrm{N}$ meridionally, in the last 10 years of the model simulation. Positive values denote eastward transport.

period in February, the LUC-driven NEUC period in July, and the MUC-driven NEUC period in November, are shown in Fig. 11. In February, the water from the east of $130^{\circ} \mathrm{E}$ section mainly flow westward (black dashed arrow in Fig. 11a), so the NEUC as well as the LUC and MUC does not exist. However, in July, the southward LUC at $18^{\circ} \mathrm{N}$ appears and fuels waters into the NKMU region from the north to form the NEUC_N (black arrows in Fig. 11b). In contrast, the NEUC_S is fed by the northward MUC from $8^{\circ} \mathrm{N}$ in November (black arrows in Fig. 11c). It is interesting to see that the NEUC_N and NEUC_S do not coexist (see Fig. 12 for more details) and there is always a weak or westward transport on the opposite side of the NEUC at least in July and November.

The seasonal variations of the net volume transports in the NEUC, LUC, and MUC show the connections among the undercurrents quantitatively (Fig. 12). The sum of the LUC net transport and the MUC net transport is almost equal to the NEUC net transport (Fig. 12a). The LUC net transport has a significant seasonal cycle, with a peak in
August/September (Fig. 12a). It varies from negative (northward) in winter to positive (southward) in summer. The MUC net transport has a relatively weak seasonality, peaking in October (Fig. 12a). By utilizing one-point mooring observation, Ren et al. (2018) showed that the MUC has a semiannual variation peaking in summer and winter. We also find similar seasonal phase of the MUC at a single longitude near the coast (not shown here). However, the seasonal variation of the meridional velocity in the MUC is longitude dependent, due to the effect of the subthermocline eddies (Zhang et al. 2014). To better identify the total contribution of the entire MUC, we used the integrated MUC net transport across the section at $8^{\circ} \mathrm{N}$. Due to the combined effect of the LUC and MUC, the NEUC net transport reaches its maximum of $\sim 9 \mathrm{~Sv}$ eastward in early autumn. The annual mean volume transports of the undercurrents suggest that the MUC is the major source of the NEUC in most time of a year, while the LUC only plays as an origin of the NEUC in summer to early autumn. 

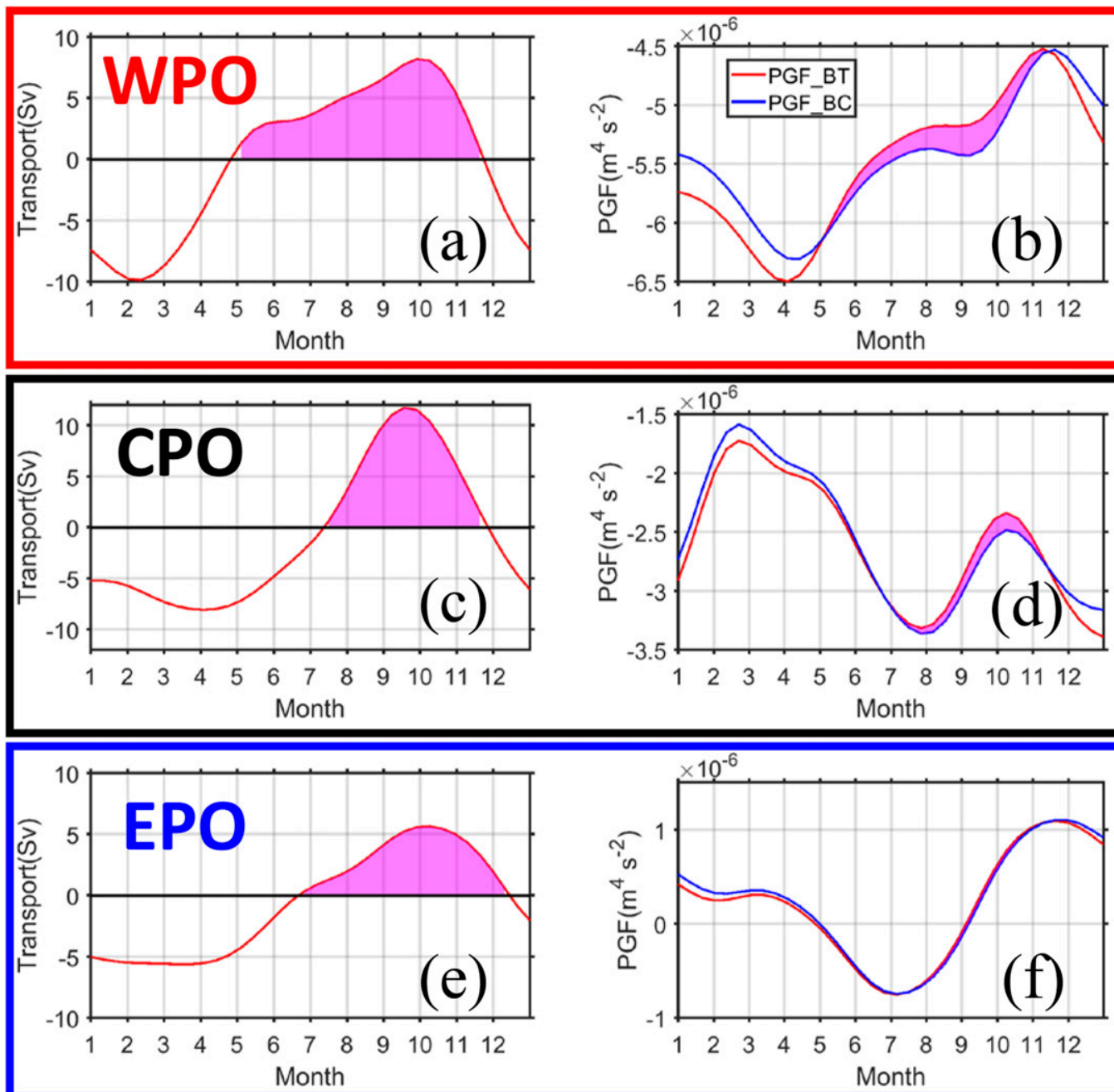

FIG. 10. (left) Seasonal variation of the NEUC net volume transport (Sv) integrated between the isopycnal surfaces of the potential densities $\left(\mathrm{kg} \mathrm{m}^{-3}\right)$ equal to 26.8 and 27.6 vertically, from $8^{\circ}$ to $18^{\circ} \mathrm{N}$ meridionally, and (right) the seasonal variation of the barotropic PGF (PGF_BT) and baroclinic PGF (PGF_BC) $\left(\mathrm{m}^{4} \mathrm{~s}^{-2}\right.$ ) averaged within the same region at (a),(b) $130^{\circ} \mathrm{E}$ (WPO), (c),(d) $180^{\circ}$ (CPO), and (e),(f) $140^{\circ} \mathrm{W}$ (EPO). Regions shaded in pink denote the appearance of the NEUC (left panels) and the NEUC-favorable PGF condition (right panels).

According to the typical flow patterns in the LUC-driven and MUC-driven periods, the NEUC has an intensified northern branch when fed by the LUC, but an intensified southern branch when fueled by the MUC (Figs. 11b,c), as is also shown in Fig. 7. We separate the NEUC net transport into the NEUC_N portion within $13^{\circ}-18^{\circ} \mathrm{N}$ and the NEUC_S portion within $8^{\circ}-13^{\circ} \mathrm{N}$. The seasonal transport and phase of the LUC coincide with the NEUC_N transport, while the MUC matches with NEUC_S (Fig. 12b). The LUC and the NEUC_N both peak in summer, and the MUC and the NEUC_S reach their maximums in late autumn. The correlation coefficient between the net transports of the LUC (MUC) and the NEUC_N (NEUC_S) in the last 10 years of the model simulation reaches $0.68(0.72)$. The dynamic linkage between the LUC/MUC and the
NEUC_N/NEUC_S has also been investigated and confirmed by using the relationship of water properties in these undercurrents (Wang et al. 2015). With the potential temperature and salinity diagrams at different sections, Wang et al. (2015) pointed out that the NEUC_N is mainly fed by the LUC with North Pacific Intermediate Water (NPIW), while the NEUC_S is largely contributed by the MUC with Antarctic Intermediate Water (AAIW) from the Southern Hemisphere.

In summary, the MUC is the main source of the NEUC_S, while the LUC only contributes to the NEUC_N in summer/autumn. During the LUC-driven NEUC period in July to September, the LUC and MUC contribute $60 \%$ and $40 \%$ of the total NEUC transport, respectively. During the MUC-driven NEUC period in late 


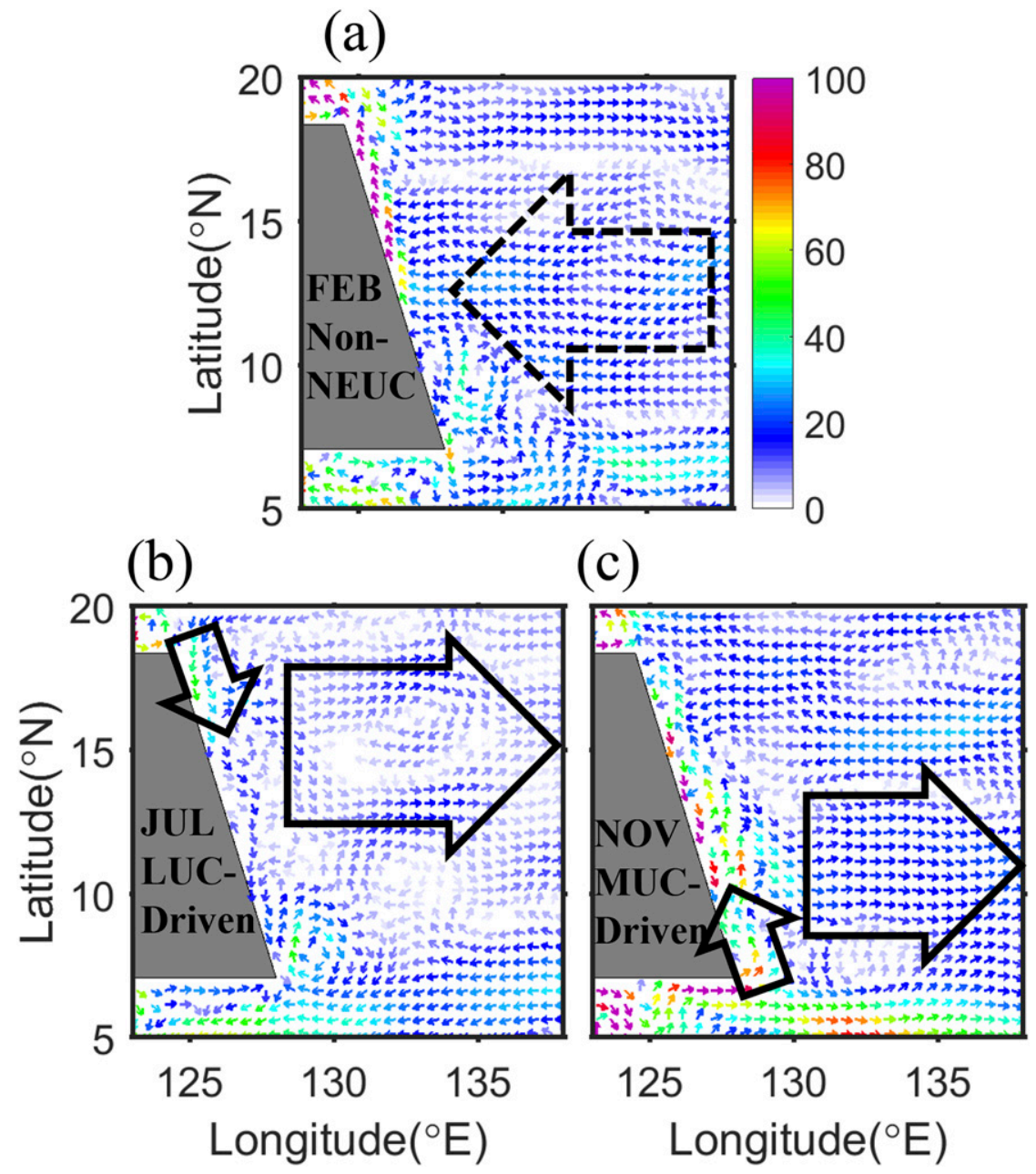

FIG. 11. Vectors of the depth-integrated velocity $\left(\mathrm{m}^{2} \mathrm{~s}^{-1}\right)$ between the isopycnal surfaces of the potential densities $\left(\mathrm{kg} \mathrm{m}^{-3}\right)$ equal to 26.8 and 27.6 in (a) February, (b) July, and (c) November. These three months represent the non-NEUC, LUC-driven NEUC, and MUC-driven NEUC periods. Black dashed arrow denotes westward current, and black solid arrows denote the LUC, MUC, NEUC_N, and NEUC_S.

autumn, the MUC contributes to almost all the NEUC. In winter to spring, the NKMU region is occupied by a wide westward current. This westward current in the non-NEUC period mainly merges to the northward LUC, while its transport at the MUC section is weak.

\section{Formation mechanism of the NEUC}

\section{a. Dynamics of influx and intrinsic response}

To identify the multiforcing processes of the lateral fluxes, bottom and top forces and intrinsic dynamics of flow and isopycnal interaction, we use a domain- and layer-integrated vorticity equation (LIVE; Gan et al. 2016) within the NKMU layer. The two isopycnal surfaces with potential densities of 26.8 and $27.6 \mathrm{~kg} \mathrm{~m}^{-3}$ are used to represent the top and bottom boundaries of the NKMU layer (green lines in Figs. $4 a-c$ ). For the horizontal domain, we select the area from the east coast of the Philippines to $130^{\circ} \mathrm{E}$ zonally and $8^{\circ}-18^{\circ} \mathrm{N}$ meridionally (black box in Fig. 2a). Following Gan et al. (2016), the LIVE can be written as

$$
\Omega_{\mathrm{acc}}=\Omega_{\mathrm{cor}}+\Omega_{\mathrm{hadv}}+\Omega_{\mathrm{pgf}}+\Omega_{\mathrm{vadv}}+\Omega_{\mathrm{vvisc}} .
$$

The left-hand side of Eq. (2), that is, the acceleration term $\Omega_{\text {acc }}$, represents the vorticity change with time. On the right-hand side, we have the divergence term $\Omega_{\text {cor }}$ and the horizontal nonlinear advection term $\Omega_{\text {hadv }}$. The term $\Omega_{\text {pgf }}$ gives the pressure torque (PT) that is induced by the curl of the horizontal force exerted by the top and bottom boundaries on the water body. The horizontal 

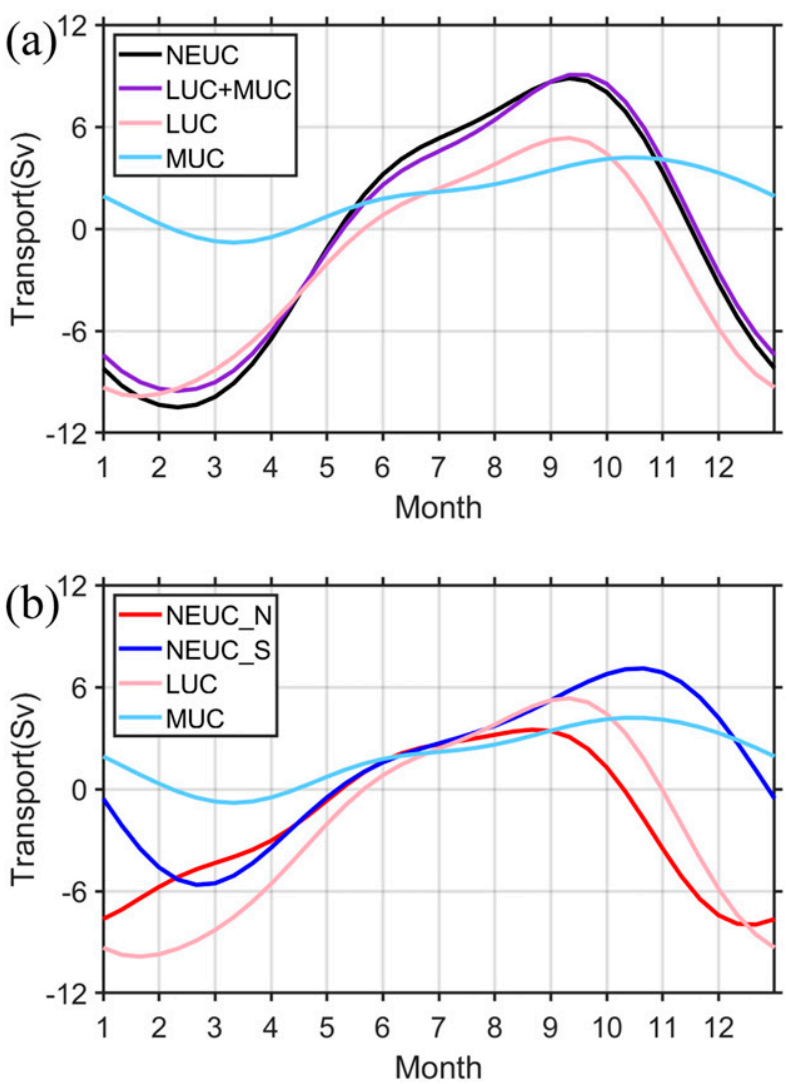

FIG. 12. Seasonal variation of the net volume transport in (a) the NEUC, the LUC, and the MUC; (b) the northern branch of the NEUC (NEUC_N), the southern branch of the NEUC (NEUC_S), the LUC, and the MUC.

gradient of the vertical velocity at the tilting isopycnal surfaces ( 26.8 and $27.6 \mathrm{~kg} \mathrm{~m}^{-3}$ ) is indicated by $\Omega_{\mathrm{vadv}}$, and the friction curl over the top and bottom of the NKMU layer is represented by $\Omega_{\mathrm{vvisc}}$. Similar to Gan et al. (2016), we apply Stokes' theorem to the $\Omega_{\text {cor }}$ term in Eq. (2) over the domain concerned:

$$
\int_{A} \nabla \times \mathbf{F} d A=\oint_{S} \mathbf{F} d S
$$

where $\mathbf{F}$ can be any vectors, such as the Coriolis term in momentum equations. Thus, we convert $\Omega_{\text {cor }}$ into the lateral fluxes of PV through the NEUC, LUC and MUC to give

$$
\begin{aligned}
\mathrm{fNEUC}= & \mathrm{fLUC}+\mathrm{fMUC}-\Omega_{\mathrm{acc}}+\Omega_{\mathrm{hadv}}+\Omega_{\mathrm{vadv}} \\
& +\Omega_{\mathrm{pgf}}+\Omega_{\mathrm{vvisc}},
\end{aligned}
$$

where fNEUC is the PV received by the NEUC, and fLUC and fMUC are the PV inputs to the NEUC from the LUC and the MUC, respectively. Equations (2) and (4) describe the dynamics in the NEUC through the linkage of extrinsic and intrinsic physical processes that govern the formation, development, and variation of the NEUC.

The fNEUC term is determined mainly by fLUC and fLUC (Fig. 13). The seasonality of fNEUC is consistent with the seasonal variation of the NEUC (Fig. 12a), and fLUC and fMUC are correlated with the LUC and MUC transports, respectively. Due to the different seasonal phases of fLUC and fMUC, the PV outflux through the NEUC originates from fLUC in late summer and from fMUC in late autumn. In August/September when fNEUC reaches its peak, fLUC contributes to $\sim 80 \%$ of fNEUC, while fMUC, $\Omega_{\mathrm{hadv}}$, and $\Omega_{\mathrm{pgf}}$ occupy the $20 \%$ remained. The fLUC term dominates the seasonal phase of the fNEUC. Also, fMUC serves as a source of the positive fNEUC during most of the year, suggesting that the MUC is a relatively stable source for the eastward NEUC waters. The $\Omega_{\text {hadv }}$ term mainly shows the advection of relative vorticity caused by the velocity shear in the coastal boundary layer. In most of the year, the NKMU circulation in the domain is energetic, so $\Omega_{\mathrm{hadv}}$ is relatively large. However, in the transient period such as May and November, the three undercurrents (NEUC, LUC and MUC) are all relatively weak. As a result, the effect of $\Omega_{\text {hadv }}$ becomes minor. Meanwhile, the vorticity acceleration $\Omega_{\text {acc }}$ approaches 0 because the circulation is in a quasi-steady state, and the horizontal gradient of the vertical velocity at the tilting isopycnal surface boundaries $\left(\Omega_{\mathrm{vadv}}\right)$ is also small, due to the weak strength of the vertical motion. The terms $\Omega_{\mathrm{pgf}}$ and $\Omega_{\mathrm{vvisc}}$, which indicate different physical processes of horizontal force curls applied to the vertical boundaries of the NKMU layer at the top and bottom isopycnal surfaces ( 26.8 and $27.6 \mathrm{~kg} \mathrm{~m}^{-3}$ ), will be discussed in the next section.

\section{b. Effect of pressure torque and stress curl}

We convert $\Omega_{\mathrm{pgf}}$ and $\Omega_{\mathrm{vvisc}}$ into the isolated forcing at the top and bottom isopycnal surfaces within the NKMU layer. The $\Omega_{\text {pgf }}$ term in Eq. (4) represents the PT at the vertical boundaries. The PT indicates the curl of the horizontal component of the force normal to the bottom (top), which is exerted by the bottom (top) boundary on the fluid (Mertz and Wright 1992). By applying the Leibniz integral rule

$$
\begin{aligned}
\frac{\partial}{\partial x}\left[\int_{H(x)}^{h(x)} F(x, z) d z\right]= & F[x, h(x)] \frac{\partial h(x)}{\partial x} \\
& -F[x, H(x)] \frac{\partial H(x)}{\partial x} \\
& +\int_{H(x)}^{h(x)} \frac{\partial F(x, z)}{\partial x} d z
\end{aligned}
$$




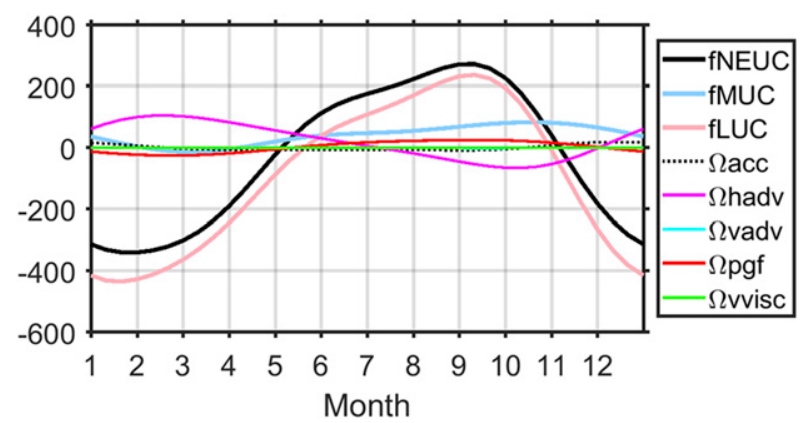

FIG. 13. Seasonal variation of the terms $\left(\mathrm{m}^{3} \mathrm{~s}^{-2}\right)$ in Eq. (4) integrated within the NKMU region.

where $h$ and $H$ represent the depth of the top and bottom isopycnal surfaces, respectively, we can expand $\Omega_{\mathrm{pgf}}$ into the components at the top and bottom boundaries as follows:

$$
\Omega_{\mathrm{pgf}}=\int_{A}[\underbrace{-\frac{1}{\rho_{0}} J\left(\mathrm{Lt}, P^{\mathrm{Lt}}\right)}_{\text {PT_TOP }} \underbrace{-\frac{1}{\rho_{0}} J\left(\mathrm{Lb}, P^{\mathrm{Lb}}\right)}_{\text {PT_BOT }}] d A,
$$

where $\mathrm{Lt}$ is the depth of the $26.8 \mathrm{~kg} \mathrm{~m}^{-3}$ isopycnal surface, representing the top of the NKMU layer, Lb is the depth of the $27.6 \mathrm{~kg} \mathrm{~m}^{-3}$ isopycnal surface, representing the bottom. The term $J$ is the Jacobean operator. The terms $P^{\mathrm{Lt}}$ and $P^{\mathrm{Lb}}$ are the pressures at the top and bottom of the NKMU layer, respectively. The two terms on the right-hand side of Eq. (6) are the PTs at the top isopycnal surface (PT_TOP) and bottom isopycnal surface (PT_BOT). Both PT_BOT and PT_TOP contribute to the seasonal variation of $\Omega_{\mathrm{pgf}}$ (Fig. 14a). PT_BOT varies from a negative value in winter to a positive one in autumn, implying that the PT at the bottom of the isopycnal surface changes largely due to the time-dependent NKMU flow pattern, including the strengths and directions of the undercurrents. In contrast, PT_TOP is mostly positive throughout the year. PT_TOP is the PT at the interface between the NKMU and the NKM, which is largely affected by the upper-layer NKM circulation. Thus, PT_TOP basically preserves its sign during a year due to the relatively steady flow pattern in the NKM. PT_BOT also shows intraseasonal variability (not shown here), which may be related to the subthermocline eddies with intraseasonal variation (Zhang et al. 2017).

Similarly, we divide $\Omega_{\text {vvisc }}$ into the stress curls (SCs) at the top (SC_TOP) and bottom (SC_BOT) boundaries of the NKMU layer as follows:
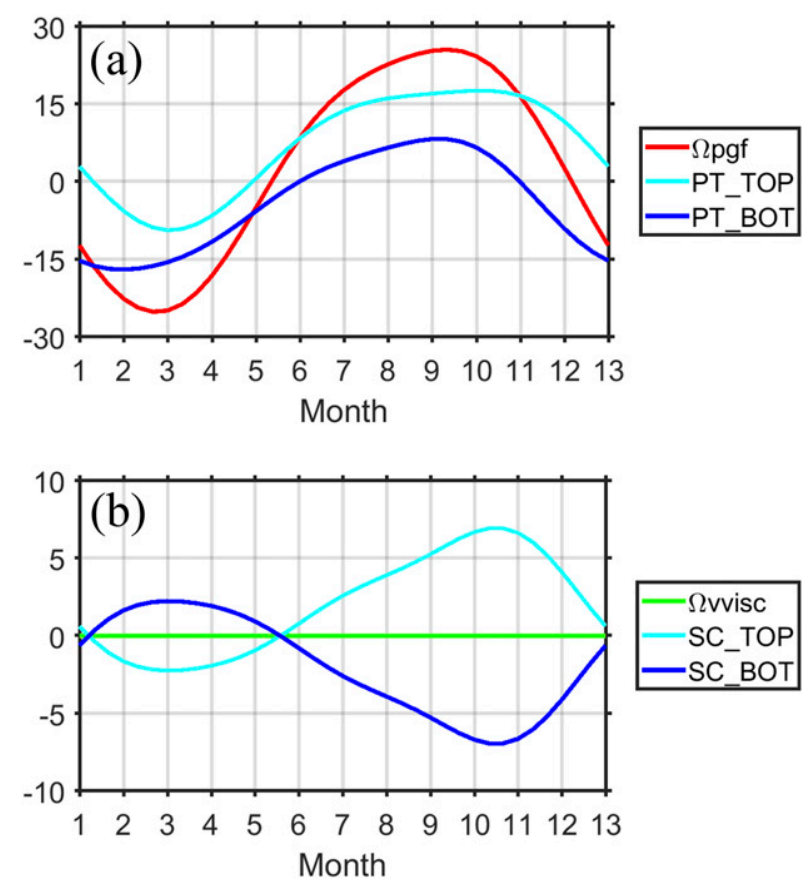

FIG. 14. Seasonal variation of (a) $\Omega_{\mathrm{pgf}}$ and (b) $\Omega_{\mathrm{vvisc}}\left(\mathrm{m}^{3} \mathrm{~s}^{-2}\right)$ with their different components in Eqs. (6) and (7), respectively.

$$
\Omega_{\text {vvisc }}=\int_{A}[\underbrace{\nabla \times \frac{\tau^{\mathrm{Lt}}}{\rho_{0}}}_{\text {SC_TOP }}+\underbrace{\nabla \times \frac{\tau^{\mathrm{Lb}}}{\rho_{0}}}_{\text {SC_BOT }}] d A,
$$

where $\tau^{\mathrm{Lt}}$ and $\tau^{\mathrm{Lb}}$ represent the stress at the top and that at the bottom of the NKMU layer, respectively. Different from the PT terms, the SC terms indicate the frictional force curls at the top and bottom boundaries, induced by the relative velocity between two layers. Compared with the other terms on the right-hand side of Eq. (2), the $\Omega_{\text {vvisc }}$ term is negligible. However, the magnitudes of SC_TOP and SC_BOT are comparable to those of PT_TOP and PT_BOT (Fig. 14). SC_TOP is positive in autumn and negative in spring, which reflects the temporal variation of the WSC averaged over the NKM region. SC_BOT compensates for SC_TOP, which implies that beneath the NKMU layer, the water is almost static. The time series of both top and bottom component in $\Omega_{\mathrm{pgf}}$ and $\Omega_{\mathrm{vvisc}}$ indicate that the horizontal torque induced by either pressure or friction reaches the peak in autumn. The deep layer below the NKMU layer dynamically affects the NEUC mainly through PT_BOT. The upper NKM layer influences the NEUC through comparable SC and PT.

The PV source of NEUC indicated by the PV outflux through the section $\left(130^{\circ} \mathrm{E}\right)$ is generally dominated by 
external forcings of lateral PV influxes carried in the LUC and MUC, and modified by intrinsic dynamics of the nonlinearity induced by the undercurrent-produced vorticity convergence and coastal-generated advection (Fig. 13). Meanwhile, the horizontal curl of the pressure force and that of the frictional force on the top and bottom boundaries of the NKMU layer are nonnegligible, in which the PT from the upper layer contributes the most (Fig. 14). We have conducted similar dynamic analysis for the NEUC in the CPO and EPO as well and drew similar conclusions: the NEUC is mainly fueled by the external forcings of lateral influxes, and is modulated and sustained by the intrinsic dynamics in the current.

\section{Discussion and summary}

Although the NEUC has been widely studied, the previous efforts mainly provided the general but spatiotemporally limited characteristics from the velocity profiles according to CTD measurement $(\mathrm{Qu}$ et al. 1998), mooring data from direct ADCP measurement (Zhang et al. 2017) and Argo float data (Yuan et al. 2014). These studies are generally based on data with a relatively low spatiotemporal resolution that limits our understanding of the detailed characteristics, physical processes, and forcing mechanisms of the NEUC. In addition, although the formation mechanisms of the NEUC alternating jets related to mesoscale variability have been identified recently (Qiu et al. 2013a,b), the origins and underlying dynamics of the large-scale eastward transport in the NEUC are seldom investigated. In this study, we investigate the characteristics and forcing mechanisms of the NEUC by conducting a processorientated numerical modeling study. The model has successfully captured the dominant features and variability of the NEUC that were either observed or reported in previous studies.

We have investigated the spatiotemporal variability of the NEUC. The NEUC is a basin-scale undercurrent that is trapped below the upper NEC between the isopycnal surfaces equal to 26.8 and $27.6 \mathrm{~kg} \mathrm{~m}^{-3}$, which is named the NKMU layer in this study. The alternating NEUC jets have well been reproduced by the idealized model. However, the widths, strengths, and number of the NEUC jets below the NEC remain uncertain from both measurements and numerical modeling, according to the previous studies. The NEUC net transport integrated within the NKMU layer reaches $\sim 9 \mathrm{~Sv}$ eastward in autumn, and gradually weakens from the WPO and CPO to the EPO. We find that the NEUC consists of a large-scale $(>400 \mathrm{~km})$ and a mesoscale $(<400 \mathrm{~km}) \mathrm{com}-$ ponent. The large-scale component has a strong seasonality and contributes $\sim 90 \%$ of the NEUC net transport, while the mesoscale component contributes only $\sim 10 \%$ due to the compensation of the alternating eastwardwestward jets. The large-scale component of the NEUC reflects mainly the effect of the meridional PGF_BC. The vertical shear of the zonal velocity induced by the meridional density gradient sustains the eastward NEUC, when PGF_BC exceeds PGF_BT in autumn. The mesoscale component of the NEUC exhibits distinct variability in different regions of the tropical North Pacific.

The NEUC has different flow patterns and branches in different seasons. In spring and winter, the eastward NEUC is absent and the waters below the NEC travel westward. In summer and autumn, the NEUC is located at the north (NEUC_N) and south (NEUC_S) of the NKMU region, respectively. The eastward NEUC mainly comes from the LUC in late summer and the MUC in late autumn. We further define three different periods according to the sources of the NEUC: the non-NEUC period from December to April, the LUC-driven NEUC period from July to September and the MUC-driven NEUC period from October to November. Due to the combined effect of the variation of the LUC and the MUC, the NEUC transport peaks in August/September. In the LUC-driven and MUC-driven periods, the LUC and the MUC mainly feed $\sim 3$ and $\sim 4 \mathrm{~Sv}$ of water into the NEUC_N and NEUC_S, respectively.

We utilize the LIVE within the water column of the NKMU to investigate the underlying dynamics of the NEUC by linking the extrinsic force to the intrinsic response in the NKMU circulation. We find that the PV influxes carried by the LUC (fLUC) and MUC (fMUC) account for large portion of the PV outflux in the NEUC (fNEUC) in different seasons. The seasonality of fLUC and fMUC is well correlated with the variation of the LUC and the MUC transport, respectively. Besides, the nonlinearity $\left(\Omega_{\text {hadv }}\right)$ due to the relative vorticity advection generated in the coastal boundary layer contributes $\sim 20 \%$ of fNEUC, when the NKMU circulation is energetic. The PT at the top and bottom boundaries of the NKMU (PT_TOP and PT_BOT) make nonnegligible contributions to the PV in the NEUC peaking in autumn. The net effect of the stress curl in the water column $\left(\Omega_{\mathrm{vvisc}}\right)$ is negligible due to the opposite influences of the stress generated by the upper-layer NKM and the static layer below the NKMU on the undercurrents system.

This study represents the first attempt to investigate the interconnection within the undercurrent system (NKMU) in the WPO both quantitatively and dynamically. We reveal the linkage between the LUC/MUC and the NEUC_N/NEUC_S, which is crucial for understanding the transports and flow patterns of the undercurrents in the WPO. The formation mechanism of the NEUC is first explored by adopting the LIVE, which 


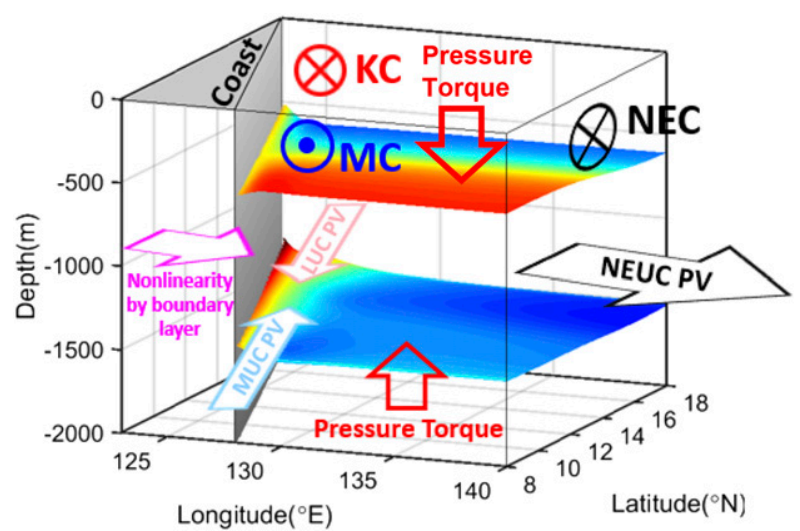

FIG. 15. Schematic of the upper NKM and lower NKMU circulation, and the main formation mechanisms of the NEUC. PV stands for planetary vorticity.

provides a novel and efficient way to isolate different physical processes. The external forcings of $\mathrm{PV}$ from the lateral fluxes (generated by the LUC and MUC, 80\%) dominates the PV outflux in the NEUC, while the coastal-induced nonlinearity $(\sim 20 \%)$ and the vertical dynamic interaction between the NKMU and the upper/deep layer (mainly induced by PT, $\sim 10 \%$ ) also contribute to the PV balance (Fig. 15). We identify the contributions of individual forcing mechanisms and fill the knowledge gap in the interconnection among the undercurrents in the NKMU system, and improve the understanding of the depth-dependent three-dimensional dynamical circulation in the WPO.

Acknowledgments. This research was supported by the key research project of the National Science Foundation of China (41930539), the National Basic Research Program of China (2015CB954004), the Research Grants Council of Hong Kong (GRF16204915 and GRF16206516), and the Center for Ocean Research (CORE), a joint research center between QNLM and HKUST. We are also grateful to the National Supercomputing Center of Tianjin and that in Guangzhou for their support. The data for this study are generated from the publically distributed Regional Ocean Model System (ROMS, https://www.myroms.org/) and are available from the corresponding author.

\section{REFERENCES}

Cravatte, S., W. S. Kessler, and F. Marin, 2012: Intermediate zonal jets in the tropical Pacific Ocean observed by Argo floats. J. Phys. Oceanogr., 42, 1475-1485, https://doi.org/10.1175/ JPO-D-11-0206.1.

Fairall, C., E. F. Bradley, J. Hare, A. Grachev, and J. Edson, 2003: Bulk parameterization of air-sea fluxes: Updates and verification for the COARE algorithm. J. Climate, 16, 571-591, https:// doi.org/10.1175/1520-0442(2003)016<0571:BPOASF >2.0.CO;2.
Firing, E., 1987: Deep zonal currents in the central equatorial Pacific. J. Mar. Res., 45, 791-812, https://doi.org/10.1357/ 002224087788327163.

Gan, J., Z. Liu, and C. R. Hui, 2016: A three-layer alternating spinning circulation in the South China Sea. J. Phys. Oceanogr., 46, 2309-2315, https://doi.org/10.1175/JPO-D-16-0044.1.

Gouriou, Y., T. Delcroix, and G. Eldin, 2006: Upper and intermediate circulation in the western equatorial Pacific Ocean in October 1999 and April 2000. Geophys. Res. Lett., 33, L10603, https://doi.org/10.1029/2006GL025941.

Hu, D., and M. Cui, 1989: The western boundary current in the farwestern Pacific Ocean. Proc. Western Pacific Int. Meeting and Workshop on TOGA COARE, Nouméa, New Caledonia, Centre Orstom de Nouméa, 123-134.

_ and - 1991: The western boundary current of the Pacific and its role in the climate. Chin. J. Oceanol. Limnol., 9, 1-14, https://doi.org/10.1007/BF02849784.

$\longrightarrow, \ldots$, T. Qu, and Y. Li, 1991: A subsurface northward current off Mindanao identified by dynamic calculation. Oceanography of Asian Marginal Seas, K. Takano, Ed., Elsevier Oceanography Series, Vol. 54, Elsevier, 359-365.

— , and Coauthors, 2013: Direct measurements of the Luzon undercurrent. J. Phys. Oceanogr., 43, 1417-1425, https://doi.org/ 10.1175/JPO-D-12-0165.1.

Jensen, T. G., 2011: Bifurcation of the Pacific North Equatorial Current in a wind-driven model: Response to climatological winds. Ocean Dyn., 61, 1329-1344, https://doi.org/10.1007/s10236-011-0427-2.

Kim, Y. Y., T. Qu, T. Jensen, T. Miyama, H. Mitsudera, H. W. Kang, and A. Ishida, 2004: Seasonal and interannual variations of the North Equatorial Current bifurcation in a high-resolution OGCM. J. Geophys. Res., 109, C03040, https://doi.org/10.1029/ 2003JC002013.

Mellor, G. L., and T. Yamada, 1982: Development of a turbulence closure model for geophysical fluid problems. Rev. Geophys. Space Phys., 20, 851-875, https://doi.org/10.1029/RG020i004p00851.

Mertz, G., and D. G. Wright, 1992: Interpretations of the JEBAR term. J. Phys. Oceanogr., 22, 301-305, https://doi.org/10.1175/ 1520-0485(1992)022<0301:IOTJT>2.0.CO;2.

Nitani, H., 1972: On the deep and bottom waters in the Japan Sea. Research in Hydrography and Oceanography, D. Shoji, Ed., Hydrographic Department of Japan Maritime Safety Agency, 151-201.

Philander, S., and R. Pacanowski, 1980: The generation of equatorial currents. J. Geophys. Res., 85, 1123-1136, https://doi.org/ 10.1029/JC085iC02p01123.

Qiu, B., and R. Lukas, 1996: Seasonal and interannual variability of the North Equatorial Current, the Mindanao Current, and the Kuroshio along the Pacific western boundary. J. Geophys. Res., 101, 12 315-12 330, https://doi.org/10.1029/95JC03204.

_ and S. Chen, 2010: Interannual-to-decadal variability in the bifurcation of the North Equatorial Current off the Philippines. J. Phys. Oceanogr., 40, 2525-2538, https://doi.org/10.1175/2010JPO4462.1.

_ _ _ , and H. Sasaki, 2013a: Generation of the North Equatorial Undercurrent jets by triad baroclinic Rossby wave interactions. J. Phys. Oceanogr., 43, 2682-2698, https://doi.org/ 10.1175/JPO-D-13-099.1.

—, D. L. Rudnick, S. Chen, and Y. Kashino, 2013b: Quasistationary North Equatorial Undercurrent jets across the tropical North Pacific Ocean. Geophys. Res. Lett., 40, $2183-$ 2187, https://doi.org/10.1002/grl.50394.

Qu, T., and R. Lukas, 2003: The bifurcation of the North Equatorial Current in the Pacific. J. Phys. Oceanogr., 33, 5-18, https://doi.org/ 10.1175/1520-0485(2003)033<0005:TBOTNE $>2.0 . C O ; 2$. 
— , H. Mitsudera, and T. Yamagata, 1998: On the western boundary currents in the Philippine Sea. J. Geophys. Res., 103, 7537-7548, https://doi.org/10.1029/98JC00263.

— T. T. Chiang, C. R. Wu, P. Dutrieux, and D. Hu, 2012: Mindanao Current/Undercurrent in an eddy-resolving GCM. J. Geophys. Res., 117, C06026, https://doi.org/10.1029/2011JC007838.

Raymond, W. H., and H. Kuo, 1984: A radiation boundary condition for multi-dimensional flows. Quart. J. Roy. Meteor. Soc., 110, 535-551, https://doi.org/10.1002/qj.49711046414.

Ren, Q., Y. Li, F. Wang, L. Song, C. Liu, and F. Zhai, 2018: Seasonality of the Mindanao Current/Undercurrent system. J. Geophys. Res. Oceans, 123, 1105-1122, https://doi.org/ 10.1002/2017JC013474.

Sakamoto, T. T., A. Sumi, S. Emori, T. Nishimura, H. Hasumi, T. Suzuki, and M. Kimoto, 2004: Far-reaching effects of the Hawaiian Islands in the CCSR/NIES/FRCGC high-resolution climate model. Geophys. Res. Lett., 31, L17212, https://doi.org/ 10.1029/2004GL020907.

Shchepetkin, A. F., and J. C. McWilliams, 2005: The Regional Oceanic Modeling System (ROMS): A split-explicit, free-surface, topography-following-coordinate oceanic model. Ocean Modell., 9, 347-404, https://doi.org/10.1016/j.ocemod.2004.08.002.

Toole, J., E. Zou, and R. Millard, 1988: On the circulation of the upper waters in the western equatorial Pacific Ocean. Deep-Sea Res., 35A, 1451-1482, https://doi.org/10.1016/0198-0149(88)90097-0.

_ R. C. Millard, Z. Wang, and S. Pu, 1990: Observations of the Pacific North Equatorial Current bifurcation at the Philippine coast. J. Phys. Oceanogr., 20, 307-318, https://doi.org/10.1175/ 1520-0485(1990)020<0307:OOTPNE $>2.0 . C O ; 2$.

Tozuka, T., T. Kagimoto, Y. Masumoto, and T. Yamagata, 2002: Simulated multiscale variations in the western tropical Pacific: The Mindanao Dome revisited. J. Phys. Oceanogr., 32, 1338-1359, https://doi.org/10.1175/1520-0485(2002)032<1338: SMVITW $>2.0 . \mathrm{CO} ; 2$.

Wang, C., 2005: Subthermocline tropical cells and equatorial subsurface countercurrents. Deep-Sea Res. I, 52, 123-135, https:// doi.org/10.1016/j.dsr.2004.08.009.

Wang, F., and D.-X. Hu, 1999: Preliminary study on the formation mechanism of counter western boundary undercurrents below the thermocline-A conceptual model. Chin. J. Oceanol. Limnol., 17, 1-9, https://doi.org/10.1007/BF02842694.

- D. $\mathrm{Hu}$, and H. Bai, 1998: Western boundary undercurrents east of the Philippines. Proc. Fourth Pacific Ocean Remote Sensing Conf. (PORSEC), Qingdao, China, PORSEC, $551-556$

— N. Zang, Y. Li, and D. Hu, 2015: On the subsurface countercurrents in the Philippine Sea. J. Geophys. Res. Oceans, 120, 131-144, https://doi.org/10.1002/2013JC009690.

Wijffels, S., E. Firing, and J. Toole, 1995: The mean structure and variability of the Mindanao Current at $8^{\circ}$ N. J. Geophys. Res., 100, 18 421-18 435, https://doi.org/10.1029/95JC01347.

Xie, S.-P., W. T. Liu, Q. Liu, and M. Nonaka, 2001: Far-reaching effects of the Hawaiian Islands on the Pacific oceanatmosphere system. Science, 292, 2057-2060, https://doi.org/ 10.1126/science.1059781.

Yaremchuk, M., and T. Qu, 2004: Seasonal variability of the largescale currents near the coast of the Philippines. J. Phys. Oceanogr., 34, 844-855, https://doi.org/10.1175/1520-0485(2004) 034<0844:SVOTLC $>2.0$. CO;2.

Yoshida, S., B. Qiu, and P. Hacker, 2010: Wind-generated eddy characteristics in the lee of the Island of Hawaii. J. Geophys. Res., 115, C03019, https://doi.org/10.1029/2009JC005417.

Yuan, D., Z. Zhang, P. C. Chu, and W. K. Dewar, 2014: Geostrophic circulation in the tropical North Pacific Ocean based on Argo profiles. J. Phys. Oceanogr., 44, 558-575, https://doi.org/10.1175/JPO-D-12-0230.1.

Zhai, F., D. Hu, and T. Qu, 2013: Decadal variations of the North Equatorial Current in the Pacific at $137^{\circ}$ E. J. Geophys. Res. Oceans, 118, 4989-5006, https://doi.org/10.1002/jgrc.20391.

Zhang, L., D. Hu, S. Hu, F. Wang, F. Wang, and D. Yuan, 2014: Mindanao Current/Undercurrent measured by a subsurface mooring. J. Geophys. Res. Oceans, 119, 3617-3628, https:// doi.org/10.1002/2013JC009693.

, F. J. Wang, Q. Wang, S. Hu, F. Wang, and D. Hu, 2017: Structure and variability of the North Equatorial Current/ Undercurrent from mooring measurements at $130^{\circ} \mathrm{E}$ in the Western Pacific. Sci. Rep., 7, 46310, https://doi.org/10.1038/ srep46310. 